\title{
Heterogeneous metasomatism in cumulate xenoliths from the Spanish Central System: implications for percolative fractional crystallization of lamprophyric melts
}

\author{
D. OREJANA \& C. VILASECA \\ Department of Petrology and Geochemistry, Complutense University of Madrid, \\ Madrid 28040, Spain (e-mail: dorejana@geo.ucm.es)
}

\begin{abstract}
The alkaline lamprophyres and diabases from the Spanish Cen System carry a heterogeneous suite of xenoliths including a group of highly altered ultramafic pyroxenites that contain $\mathrm{Cr}-\mathrm{Mg}$-rich high-T hydrous minerals (Ti-phlogopite and pargasitic to kaersuritic amphibole), indicative of modal metasomatism. The element mineral compositions of these xenoliths show three patterns: type A xenoliths, with light rare earth element enriched clinopyroxenes with high field strength element (HFSE) negative anomalies; type B xenoliths, with clinopyroxenes and amphiboles with high incompa ble element contents (large ion lithophile elements (LILE), HFSE and REE); type C xenoliths, with relatively REE- and HFSE-poor clinopyroxenes and amphiboles. These metasomatic signatures suggest the involvement of three different metasomatic agents: carbonate, silicate and hydrous fluids or melts, respectively. These agents could have been derived from the progressive differentiation of a $\mathrm{CO}_{2}-\mathrm{H}_{2} \mathrm{O}$-rich highly alkaline magma, genetically related to the Late Pennian alkaline magmatism. Because of the original sub-alk-aline nature of the pyroxenitexenoliths, they might have beenforme originally as pyroxene-rich cumulates associate with underplated Hercynian calc-alkaline basic magmas. Metasomatism as a result of the infil tration of alk-ane magmas withis these cumulates might explain the relaively highradiogenic $\mathrm{Nd}$ composition of the altere ulwamafic xenoliths.
\end{abstract}

Ultramafic xeneliths carried by mantle-derived alkaline volcanic and subvolcanic magmas sometimes include pyrexenitic types, which are much less abundant than typical mantle lithelegies (peridotites). Several possible origins have been propesed for pyroxenite xenøliths: (1) cumulates or segregations from mantle-derived magmas intrduced as dykes or veins within the lithespheric mantle (e.g. Frey \& Prinz 1978; Downes 2001); (2) cumulates crystallized within deep magma chambers near the upper mantle-lewer crust boundary, ass ciated with underplating of mafic magmas (e.g. Féménias et al. 2003); (3) restites produced during melting and genetically associated with overlying or genic bathøliths (Ducea \& Saleeby 1998); (4) remnants of oceanic crust, subducted int the asthenesphere and then streaked -ut by mantle convection until they were incorperated int the lith sphere (Allègre \& Turc tte 1986). Mørever, søme pyr xenites have been subjected to a later enrichment precess (Garride \& Bedinier 1999; Litasøv et al. 2000; Xu 2002).

The Upper Permian alkaline lamprophyres and diabases from the Spanish Central System (SCS) carry a heterøgeneous xenølith suite, including lower crustal granulites (Villaseca et al. 1999) and ultramafic pyrexenites and hornblendites (Orejana et al. 2006). Some types of pyroxenites have never been studied because of their scarcity and alteration. These altered pyrexenites show a relict high- $T$ paragenesis compesed of clinøpyr

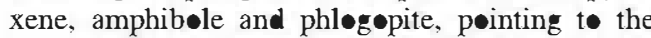
invelvement of a metas matic transformation at depth.

The nature of the metas matizing agents in enrichment processes can be breadly divided int three categories: (1) silicate melts (e.g. Ionov et al. 2002 a; Witt-Eickschen et al. 2003); (2) carbonate or carbenatitic melts and/or fluids (e.g. I॰nev 1998; Yaxley et al. 1998; Xu et al. 2003); (3) hydrøus fluids (e.g. Jøhnsøn et al. 1996).

The presence of multiple types of gechemical patterns in a single suite of ultramafic xenoliths is relatively common and sometimes has led to the conclusion that various genetically unrelated liquids were invelved (Grégøire et al. 2003; Witt-Eickschen et al. 2003), whereas, in other cases, it has been interpreted as being derived from different metasmatic agents genetically related to a single original melt by percelative fractional crystallization (Litasev et al. 2000; Iøn v et al. 2002 a; Xu \& Bodinier 2004). The widely accepted porøus melt fløw mødel (Nav॰n \& Stelper 1987) predicts the possibility of generation - f volatile-rich $\left(\mathrm{H}_{2}-\mathrm{CO}_{2}\right)$ melts or fluids frøm a single velatile-rich magma (Downes 2001; Xu \& Bodinier 2004) as a result of a continuous reaction with the rock matrix. These løw-viscesity agents 
are capable of prøducing a strøng interaction with the wall røck.

This paper focuses $\bullet$ the petrøgraphy and the majør and trace element mineral compesition of the altered pyroxenite SCS xen-liths, with the intention of constraining their origin, and discusses the interaction with metas matic agents and their relatienship with the host alkaline basic magmas at different emplacement levels.

\section{Geological background}

The SCS is a plutonic-metamorphic terrane composed of several Hercynian granitic intrusions,

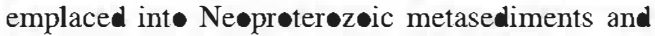
Palaeøzoic •rthøgneisses (Fig. 1). This basement

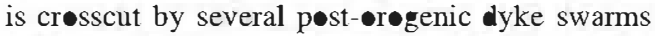
with contrasting gechemical affinities: calcalkaline, shøshønitic, alkaline and thøleiitic (Villaseca et al. 2004). The alkaline suite can be divided in tw gr $\bullet$ ups: (1) basic t॰ ultrabasic lamprophyres (camptønites) and diabases; (2) red

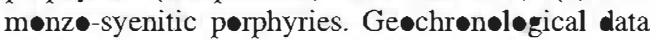
-n this alkaline magmatism yield an age of intrusion in the range $252-264 \mathrm{Ma}$ (264 Ma, $\mathrm{Ar}-\mathrm{Ar}$ in amphibøle, Perini et al. 2004; Scarrøw et al. 2006; -r $252 \mathrm{Ma}, \mathrm{U}-\mathrm{Pb}$ in zircen, FemándezSuárez et al. 2006).

The SCS alkaline magmatism occurred in an anøregenic setting during the initial stages of North Atlantic opening. Orøgenic Hercynian metamørphism in the area reached its peak at around $330 \mathrm{Ma}$ and afterwards experienced a marked retr -

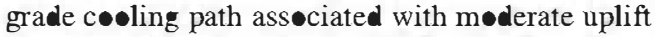
(Escuder Viruete et al. 1998). During these

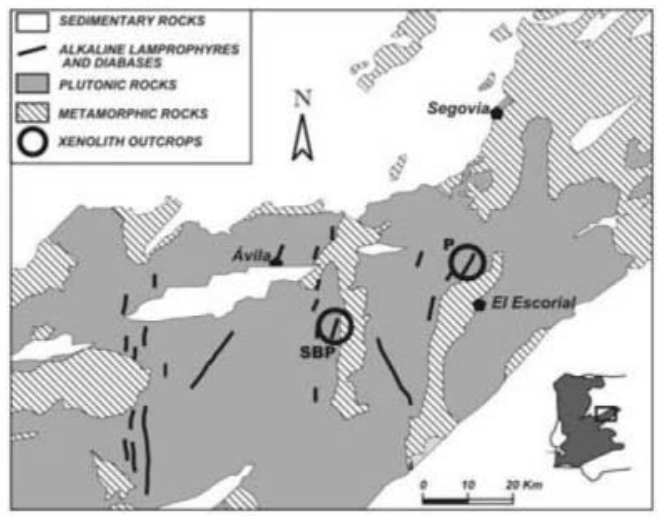

Fig. 1. Sketch map of the Spanish Cen wal System showing the location of the Permian alkaline lamprophyres and diabases, and the outcrops of the altere ulwamafic xenoliths (P, Peguerinos; SBP, San Bartolomé de Pinares).

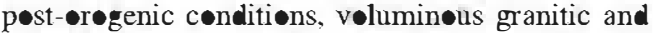
calc-alkaline magmas intruded these crustal levels,

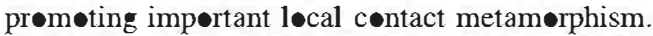
At $70 \mathrm{Ma}$ after the regional metamørphism, during Late Permian times, lamprophyre dyke swarms were emplaced at very shalløw and cœol levels, although this did not result in contact metamerphism because of the small volume of magma. These uplifted crustal bløcks int $\bullet$ which the lampr phyres were intruded have not experienced burial since Early Mesøoic times, as revealed by fissiontrack data (Bruijne \& Andriessen 2000). Møre ver,

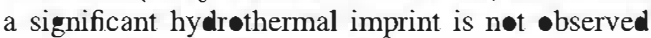
in either the lamprophyre dykes or the plutenmetamørphic wall rocks, suggesting løng-term stable crustal conditions after the alkaline magmatism. Therefore, the significant degree of alteration shøwn by the studied pyrexenite xeneliths suggests a genetic relationship with the host lamprophyric melt as discussed belew.

The SCS alkaline lamprophyres and diabases carry a wide variety $\bullet$ xen lithic material, comprising wall-røck fragments (granites and gmeises), lower crustal xenoliths (granulites sensu lato) and mafic to ultramafic pyrexene-rich cumulates from the upper mantle-lewer crust boundary. This last group of xenøliths has been divided, in previøus studies, in føur subgrøups according to their petr•graphic characteristics (Ore jana et al. 2006): (1) highly altered xen॰liths; (2) spinel pyrøxenites; (3) hydrated clinepyrexenites; (4) magmatic

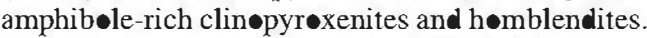

Type 2 spinel pyrexenites and type 4 hømblendites are generally larger in size and more abundant than the $\bullet$ ther ultramafic xeneliths (Orejana et al. 2006). They have been interpreted as magmatic cumulates or segregates crystallized at the upper mantle-løwer crust bøundary. The amphibøle-rich type 4 xen॰liths show magmatic textures, and have been interpreted as crystallizing from the alkaline melts (Ore jana et al. 2006). In contrast, type 2 xenøliths display a granøblastic texture and,

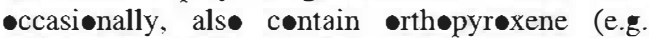
websterite 102131). Thus, they have been interpreted as being related t• earlier magmatic events ass ciated with underplating of calc-alkaline basic magmas at the base of the crust (Orejana et al. 2006).

Types 1 and 3 ultramafic xen liths of Orejana et al. (2006) show a variable amount of high- $T$

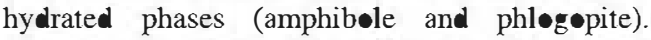
Nevertheless, their crystalløblastic fabric and modal mineral composition differentiate them frøm the clearly igmeous-textured type 4 xen॰liths

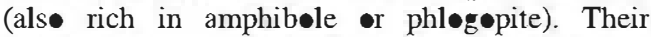
scarcity and small size represent majør hindrances to their study, especially when considering their whole-røck and isøtope geochemistry. 
Nevertheless, in this paper we present a general petregraphic and gechemical characterization of these enclaves, mainly focusing on major and trace element mineral composition, although whøle-rock geochemical analyses and $\mathrm{Sr}-\mathrm{Nd}$ is tope determinations have been carried out on tw॰ samples.

\section{Analytical methods}

The majør element mineral compesition has been analysed at the Centr• de Micrøscøpía Electrónica 'Luis Bru' (Cømplutense University of Madrid) using a Jeøl JZA-8900 M electrøn micrøpr be with four wavelength-dispersive spectrømeters. Analytical conditions were an accelerating voltage -f $15 \mathrm{kV}$ and an electron beam current of $20 \mathrm{nA}$, with a beam diameter of $5 \mu \mathrm{m}$. Elements were counted for $10 \mathrm{~s}$ on the peak and $5 \mathrm{~s}$ on each background position. Corrections were made using the ZAF methed.

We have determined in situ the concentrations -f 27 trace elements (rare earth elements (REE), $\mathbf{B a}, \mathbf{R b}, \mathrm{Tb}, \mathrm{U}, \mathrm{Nb}, \mathrm{Ta}, \mathrm{Pb}, \mathrm{Sr}, \mathrm{Zr}, \mathrm{Hf}, \mathrm{Y}, \mathrm{V}, \mathrm{Cr}$ and $\mathrm{Ni}$ ) in clinepyrexene, amphibøle and phlegepite, $\bullet>130 \mu \mathrm{m}$ thick pølished sections by laser ablation inductively coupled plasma mass spectrømetry (LA-ICP-MS) at the University $\bullet$ Brist $\bullet$ using a VG Elemental PlasmaQuad 3 ICP-MS system coupled t• a VG LaserPrøbe II $(266 \mathrm{~nm}$ frequency-quadrupled Nd-YAG laser). The counting time for $\bullet$ ene analysis was typically $100 \mathrm{~s}(40 \mathrm{~s}$ measuring as blank to establish the background and $60 \mathrm{~s}$ for the remainder of the analysis). The diameter of laser beam was around $20 \mathrm{~m}$. The NIST 610 and 612 glass standards were used to calibrate relative element sensitivities for the analyses -f the silicate minerals. Each analysis was nørmalized to $\mathrm{Ca}$ using concentrations determined by electrøn micrøpr be.

The whøle-røck majør and trace element cømpsition of tw xenoliths was analysed at the CNRS-CRPG Nancy. The samples were melted using $\mathrm{LiBO}_{2}$ and disselved with $\mathrm{HNO}_{3}$. Selutions were analysed by inductively coupled plasma at॰mic emission spectrømetry (ICP-AES) før major elements, whereas trace elements were determine by ICP-MS. Uncertainties in majer elements are bracketed between 1 and $3 \%$, except for Mn $(5-10 \%)$ and $\mathbf{P}_{2} \mathbf{O}_{5}(>10 \%)$. Carignan et al. (2001) have evaluated the precision of Nancy ICP-MS analyses at low concentration levels from repeated analyses of the international standards BR, DR-N, UB-N, AN-G and GH. The precision for $\mathbf{R b}, \mathrm{Sr}, \mathrm{Zr}, \mathrm{Y}, \mathrm{V}, \mathrm{Hf}$ and most of the REE are in the range $1-5 \%$, whereas they range from 5 to $10 \%$ for the $\bullet$ ther trace elements, including $\mathrm{Tm}$.
Møre information on the procedure, precision and accuracy of Nancy ICP-MS analyses has been given by Carignan et al. (2001).

$\mathrm{Sr}-\mathrm{Nd}$ is $\bullet$ pic analyses $\bullet \mathrm{tw} \bullet \mathrm{xen} \bullet \mathrm{liths}$ were

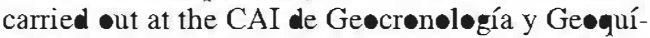
mica Isøópica of the Complutense University of Madrid, using an aut•mated VG Sectør 54 multic lector thermal ionization mass spectrømeter with data acquired in multidynamic mode. Is •t॰pic rati of $\mathrm{Sr}$ and Nd were measured on a subset of whøle-røck pøwders. The analytical prøcedures used in this laboratery have been described elsewhere (Reyes et al. 1997). Repeated analysis - NBS 987 gave ${ }^{87} \mathrm{Sr} /{ }^{86} \mathrm{Sr}=\mathbf{0 . 7 1 0 2 4 9} \pm 30(2 \sigma$, $n=15)$ and for the JM Nd standard ${ }^{143} \mathrm{Nd} /{ }^{144}$ $\mathbf{N d}=0.511809 \pm 20(2 \sigma, n=13)$. The $2 \sigma$ err $\bullet$ on $\varepsilon(\mathrm{Nd})$ calculation is \pm . An estimated age of $265 \mathrm{Ma}$ was used for calculating initial is tøpic raties.

\section{Petrography of xenoliths}

Although the altered pyroxenite xenolith suite has been previously classified int tw types, the similarity of their petrography (high degree of alteration and the common presence of hightemperature hydrated minerals), suggests that they all form a heterogeneous, genetically related group. Thus, for the sake of simplicity, we shall refer to them hereafter as altered ultramafic SCS xeneliths.

These altered ultramafic SCS xeneliths have

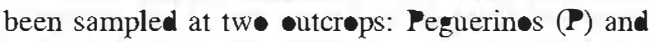
San Bartølømé de Pinares (SBP) (Fig. 1). They are small in size, never exceeding $2.7 \mathrm{~cm}$, and are irregular to semi-rounded in shape. Their modal compesition is summarize in Table 1.

SCS altered pyrexenite xeneliths are compesed

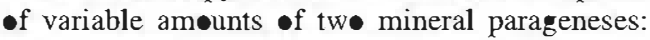
(1) a granıblastic high- $T$ assemblage of clinøpyrex-

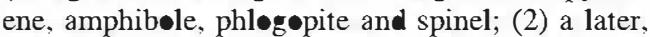
volatile-rich alteration assemblage of chlorite group and talc minerals that pseudomerph granblastic crystals. The texture of the altere ultramafic SCS xeneliths is fine-grained, equigranular and granıblastic, shøwing typical triple junctions indicative of solid-state equilibration. This high- $T$ crystalløblastic fabric is preserved irrespective of the degree of secondary alteration.

Mineral heterogeneity might be related to the small size of these xenoliths, giving rise to a wide

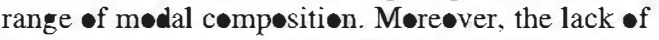
-livine suggests a cumulate origin instead of representing mantle fragments. Primary high- $T$

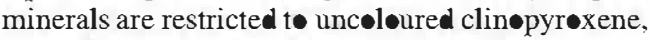
pargasitic to kaersutitic amphibøle, Ti-phløg•pite

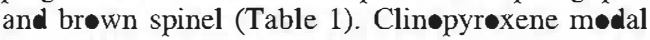




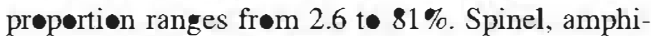

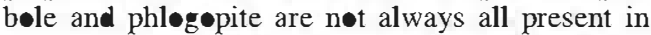
these xenoliths. Although spinel is regularly disse-

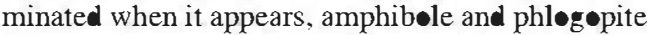
are møre dispersed. Neither mineral zøning n॰r pyr-xene exsølution lamellae have been $\bullet$ bserved in these high- $T$ minerals.

These pyroxenite xen-liths have secondary minerals in variable prøportions from 19 t॰ $95 \%$. These alteration phases are mainly talc and chlorite group minerals, pale brownish chlorite being the most cømmøn. Unaltered ørthøyrexene has never been -bserved in these xenoliths. Olivine pseudemorphs are unlikely because the presence of this mineral in the original paragenesis is very limited according to their normative composition as deduced from

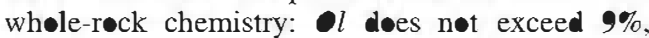
whereas Hy ranges from 80 to $99 \%$ (see Table 4). The normative compesitions of analysed samples plot within the field of orth $\bullet y$ rexenite in the ultramafic rocks classification diagram, pøinting to -rthøpyrexene as the main original mafic phase (n॰w pseudomørphed) in the modal compesition -f these xenoliths. In summary, normative and modal compesitions pøint to a wide range of

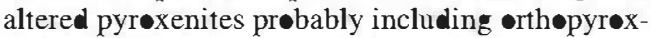
enites and websterites.

The mineral chemistry of chlorite pseudomørphs alsø supports the interpretation that $\bullet$ rthopyroxene was the main primary mineral of these pyr $\bullet$ xenite xenøliths. When comparing the chemical compsition of the abundant chloritized pseudomorphs with secøndary chlørite from the partial replacement $\bullet$ f $\bullet$ th $\bullet y$ r $\bullet$ xene fr $\bullet$ the websterite sample 102131 (type 2 xenoliths of Orejana et al. 2006)

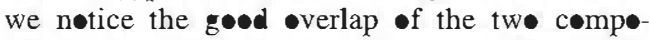
sitional fields (Fig. 2). Furthermore, the pale brownish colour and the good cleavage of both chlorite crystals reinforce the suggestion that they belong to the same chlorite type. The wide chemical composition shøwn by these chlorites suggests complex chemical substitution precesses towards talc endmember compesitions (Fig. 2). Talc and •ccasional $\mathrm{Fe}$-sulphide are $\bullet$ ther secondary mineral phases.

\section{Mineral chemistry}

Acc $\bullet$ rding t mineral chemistry, we have classified the altered xen-liths int three sub-types by their trace element contents (mainly light rare earth elements (LREE) in clinopyroxene and amphibøle): type A xenøliths, with Sr-LREE-enriched clinpyroxene and lacking high- $T$ hydrous phases

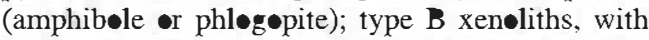
LREE-enriched clinøpyroxene and amphibøle; and

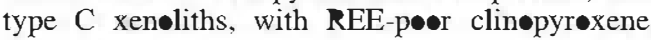
and amphibøle. 


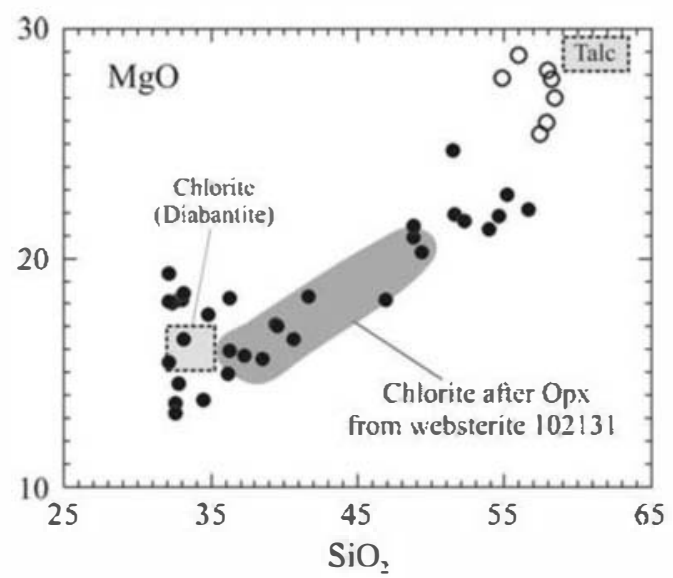

Fig. 2. Major element contents of secondary alteration minerals of the ul wamafic SCS xenoliths (-, brownish chlorite $\bullet$, talc). Talc and chlorite (diabante) compositional fields taken from data summarized by Deer et al. (1976).

\section{Major elements}

Clinøpyrexene is diopside and augite with a heter geneous major and trace element composition (Tables 2 and 3). $\mathrm{Al}_{2} \boldsymbol{}_{3}, \mathrm{Ti}_{2}$ and $\mathrm{Na}_{2} \bullet$ may reach high concentrations: $4.2-7.7 \mathrm{wt} \%, 0.3-$ $1.4 \mathrm{wt} \%$ and $0.41-1.75 \mathrm{wt} \%$, respectively. Nevertheless, the heter geneity shøwn by the majør element compesition highlights the large comp•sitional ranges shøwn by clinøpyrexenes frøm type B xeneliths (Fig. 3). This latter group has Mg-number values ranging frøm 0.78 to $\mathbf{0 . 9 3}$, whereas types $\mathrm{A}$ and $\mathrm{C}$ are restricted to

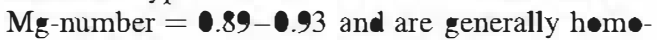

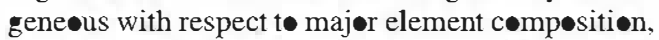
except før Na (Fig. 3).

Amphibele is absent in type A xenoliths

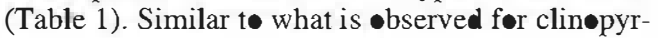

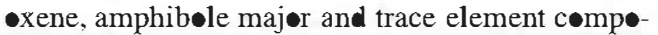
sition is heter geneous for type B xen॰liths (Fig. 3). On the whøle, they are $\mathrm{Ti}-\mathrm{Al}$-rich pargasites or kaersutites (Ti ${ }_{2}: 1.9-5.1 \mathrm{wt} \% ; \mathrm{Al}_{2} \Theta_{3}: 12.9-$ $15.7 \mathrm{wt} \%$ ), with $\mathrm{Na}_{2}$ in the range $2.2-3.3 \mathrm{wt} \%$, $\mathrm{K}_{2} \bullet$ frøm $\bullet .71$ t॰ $1.84 \mathrm{wt} \%$ and high $\mathrm{Mg}$-number

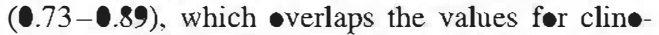

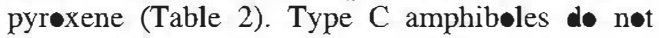
differ significantly in their majer element compsition from type B (Fig. 3), but have higher $\mathrm{Cr}$ contents (7436-7845 ppm, compared with 20043236 ppm før type B; Table 3 ).

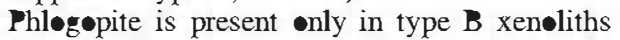
(Table 1). Its majer element compesition is hømøgeneøu, with $\mathrm{Mg}$-number ranging frøm 0.82 t $\bullet .86$, •verlapping the values for clinøpyroxene and amphibøle (Table 2). They typically have high $\mathrm{Ti}_{2}(2.46-5.14 \mathrm{wt} \%), \mathrm{Cr}_{2}$ $(0.42-1.11 \mathrm{wt} \%)$ and $\mathrm{Al}_{2} \mathrm{O}_{3}(15.6-16.8 \mathrm{wt} \%)$ contents (Fig. 3). This compesition contrasts with the lower $\mathrm{Mg}$-number and $\mathrm{Cr}$ contents, and higher Ti concentrations of phlogøpites frøm the SCS hornblenditic xenoliths, which have been interpreted as magmatic segregates genetically linked to the host alkaline melts (Orejana et al.

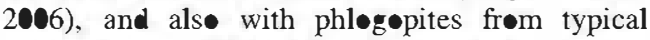
cumulate xeneliths (Fig. 3); but it is very

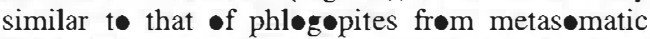
ultramafic xenoliths, such as these reperted by Shaw (2004). Because of the scarcity and small

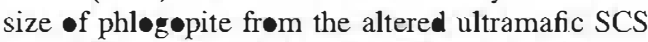
xeneliths, we could not analyse their trace element compesition.

Spinel is present in the three altered ultramafic SCS xenolith types. It is always $\mathrm{Al}-\mathrm{Cr}$-rich and

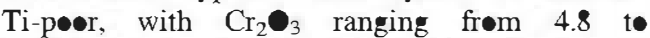
$14.3 \mathrm{wt} \%, \mathrm{Al}_{2} \bullet_{3}$ frøm 49.7 t $63.5 \mathrm{wt} \%, \mathrm{Ti} \boldsymbol{O}_{2}$ $<.22 \mathrm{wt} \%$ and $\mathrm{Mg}$-number from $\bullet .69$ to 0.79 (Table 2). Nevertheless, spinel from type $\mathbf{B}$ xen $\bullet$ liths shøws the lowest $\mathrm{Cr}_{2} \mathbf{O}_{3}$ and highest $\mathrm{Al}_{2} \mathbf{}_{3}$ concentrations (4.8 and $63.5 \mathrm{wt} \%$, respectively). Spinel of type $\mathrm{A}$ and $\mathrm{C}$ xenoliths displays the same narr $\mathrm{w}$ compesitional range: $11.3-14.3 \mathrm{wt} \%$ for $\mathrm{Cr}_{2} \mathrm{O}_{3}$ and $49.7-55.9 \mathrm{wt} \%$ for $\mathrm{Al}_{2} \mathrm{O}_{3}$.

\section{Trace elements}

Clinøpyrexene trace element characteristics emphasize the existence of three types of altered ultramafic xeneliths (Fig. 4a). Clinepyrexenes from type A xenøliths display the mest LREE-enriched patterns, which may reach 100 times chøndrite values, whereas heavy REE (HREE) tend to converge to concentrations shøw

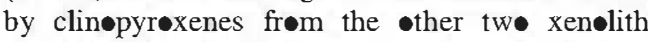
types. This LREE enrichment is als accompanied by high contents of $\mathrm{Sr}(208-230 \mathrm{ppm})$, but low values for $\mathrm{Zr} \quad(22-26 \mathrm{ppm})$ and $\mathrm{Nb} \quad(.12-$ $0.24 \mathrm{ppm}$ ) (Table 3). Accordingly, their primitive mantle-normalized trace element pattern is distinctive, with strong negative anımalies at $\mathrm{Zr}$ - $\mathrm{Hf}$ and $\mathrm{Nb}-\mathrm{Ta}$, and at $\mathrm{Ba}$ and $\mathrm{Ti}$ (Fig. 4b). Type B clinøpyrexenes are als enriched in LREE, but with løwer concentrations than type A. Møreøver, they yield convex-upwards chondrite-normalized REE patterns with the peak position between Ce and Nd, which is characteristic of clinøpyroxenes formed as deep cumulates crystallized from basic alkaline magmas (Irving \& Frey 1984) (Fig. 4a). Nevertheless, the LREE concentrations of type $\mathbf{B}$ clinøpyrexenes are significantly higher than these shown by Irving \& Frey (1984). The primitive mantle-normalized trace element pattern of these clinøpyrexenes is very similar to that of type A, but concentrations of $\mathrm{Zr}(32.1-72.5 \mathrm{ppm})$, Hf 
Table 2. Major element composition and $\mathbf{P}-\mathrm{T}$ estimates of representative high-T minerals from the SCS altered ultramafic xenoliths

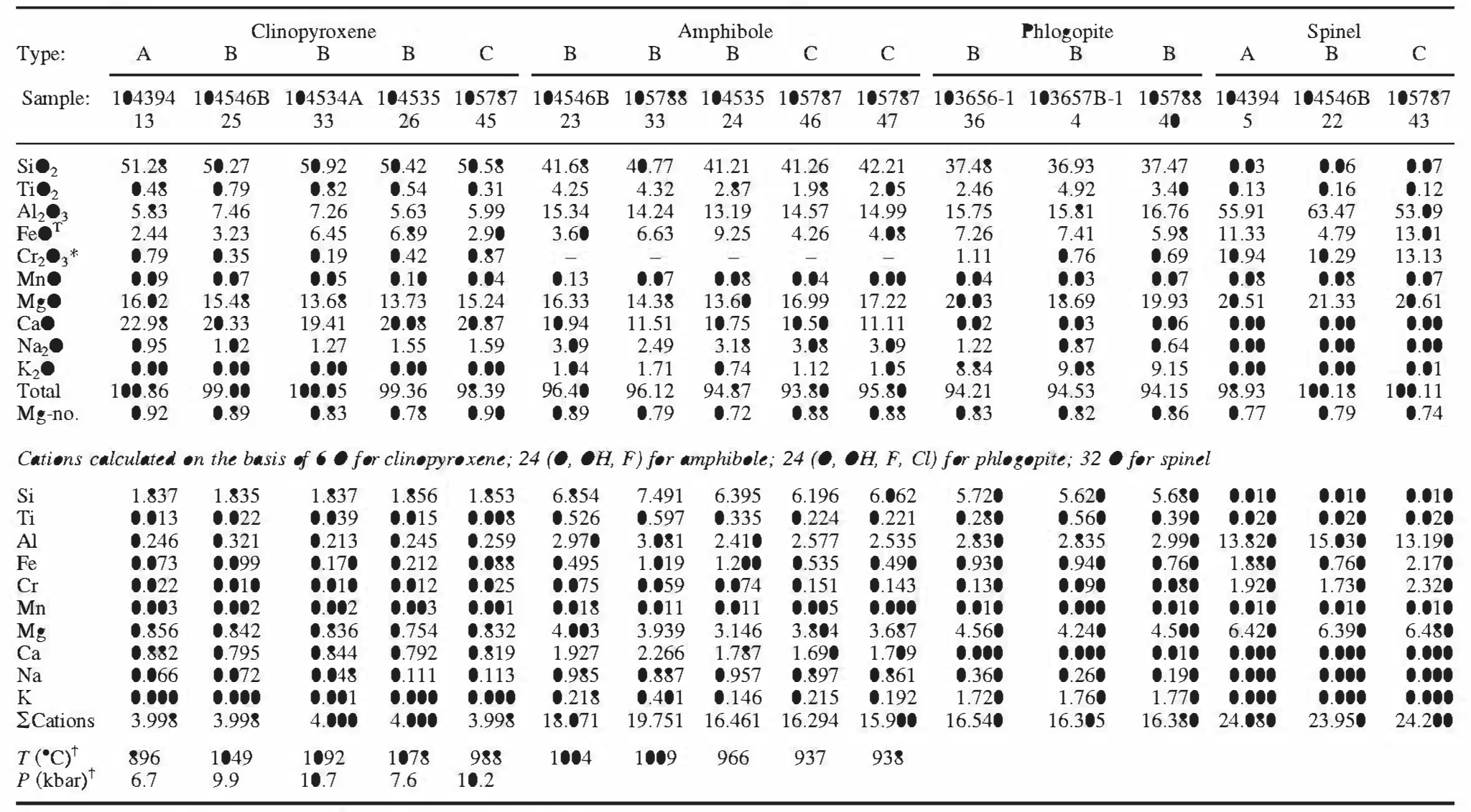

*Cr contents in amphibole have been determined by LA-ICP-MS (see Table 3).

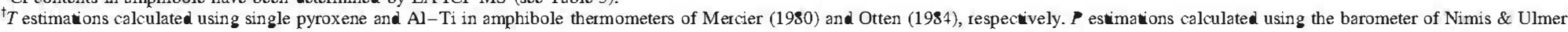
(1998). 
Table 3. Trace element composition of representative clinopyroxenes and amphiboles from SCS altered ultramafic xenoliths

\begin{tabular}{|c|c|c|c|c|c|c|c|c|c|c|c|c|c|c|c|c|c|}
\hline \multirow{4}{*}{$\begin{array}{l}\text { Type: } \\
\text { Sample: }\end{array}$} & \multicolumn{9}{|c|}{ Clinopyroxene } & \multicolumn{8}{|c|}{ Amphibole } \\
\hline & A & A & A & B & B & B & $\mathrm{C}$ & $\mathrm{C}$ & $\mathrm{C}$ & B & B & B & B & B & $\mathrm{C}$ & $\mathrm{C}$ & $\mathrm{C}$ \\
\hline & 104394 & 104394 & 104394 & $104546 \mathrm{~B}$ & 104535 & $104534 \mathrm{~A}$ & 105787 & 105787 & 105787 & $104546 \mathrm{~B}$ & $104546 \mathrm{~B}$ & $104534 \mathrm{~A}$ & $104534 \mathrm{~A}$ & $104534 \mathrm{~A}$ & 105787 & 105787 & 105787 \\
\hline & 12 & 21 & 51 & 41 & 33 & 41 & 10 & 7 & 9 & 31 & 33 & 22 & 23 & 24 & 1 & 2 & 3 \\
\hline $\mathrm{Ba}$ & 0.84 & 1.37 & 2.42 & 1.57 & 0.92 & 3.92 & 2.60 & 2.17 & $\mathrm{bd}$ & 172 & 178 & 236 & 247 & 228 & 79.7 & 82.1 & 84.3 \\
\hline $\mathrm{Rb}$ & $\mathrm{bd}$ & $\mathrm{bd}$ & 1.65 & $\mathrm{bd}$ & 0.67 & 0.43 & 0.65 & 0.47 & $\mathrm{bd}$ & 4.84 & 5.12 & 6.01 & 6.03 & 6.20 & 5.69 & 5.97 & 6.33 \\
\hline $\mathrm{Sr}$ & 230 & 212 & 215 & 95.3 & 107.6 & 101.2 & 44.8 & 44.0 & 40.9 & 278 & 208 & 474 & 488 & 504 & 12 & 120 & 117 \\
\hline $\mathrm{Pb}$ & na & na & na & na & na & na & 0.46 & 0.16 & - 17 & na & na & na & na & na & 0.29 & 0.45 & 0.43 \\
\hline Th & 0.06 & 0.10 & 0.08 & 0.31 & 0.24 & 0.14 & $\mathrm{bd}$ & $\mathrm{bd}$ & 0.06 & 0.20 & 0.33 & 0.15 & 0.16 & อ. 19 & $\mathrm{bd}$ & $\mathrm{bd}$ & $\mathrm{bd}$ \\
\hline $\mathrm{U}$ & $\mathrm{bd}$ & $\mathrm{bd}$ & 0.03 & 0.09 & 0.08 & 0.05 & $\mathrm{bd}$ & $\mathrm{bd}$ & $\mathrm{bd}$ & 0.05 & 0.11 & 0.03 & 0.05 & 0.04 & $\mathrm{bd}$ & bd & $\mathrm{bd}$ \\
\hline $\mathrm{Zr}$ & 24.5 & 24.8 & 25.9 & 38.4 & 70.5 & 56.2 & 17.5 & 16.7 & 16.4 & 66.7 & 73.2 & 52.6 & 51.8 & 52.6 & 16.8 & 18.0 & 15.6 \\
\hline $\mathrm{Nb}$ & 0.10 & 0.17 & 0.13 & 1.56 & 0.27 & 0.59 & .16 & $\mathrm{bd}$ & 0.11 & 78.4 & 53.2 & 43.1 & 45.3 & 45.2 & 1.35 & 1.23 & 1.36 \\
\hline$Y$ & 16.8 & 15.5 & 14.9 & 19.9 & 24.4 & 18.6 & 16.7 & 15.5 & 15.1 & 21.7 & 21.8 & 21.7 & 22.7 & 21.8 & 17.3 & 18.2 & 17.8 \\
\hline V & 254 & 246 & 251 & 286 & 623 & 709 & 344 & 320 & 319 & 440 & 450 & 909 & 944 & 905 & 501 & 545 & 543 \\
\hline $\mathrm{Ni}$ & 393 & 404 & 386 & 315 & 116 & 74 & 432 & 286 & 325 & 605 & 596 & 129 & 149 & 129 & na & na & na \\
\hline $\mathrm{Cr}$ & 5215 & 4948 & 5020 & 2046 & 2892 & 1745 & 5239 & 4687 & 5310 & 3124 & 3236 & 2087 & 2182 & 2118 & 7436 & 7845 & 7721 \\
\hline $\mathrm{Ta}$ & 0.01 & 0.04 & 0.02 & 0.09 & 0.07 & 0.11 & 0.05 & $\mathrm{bd}$ & $\mathrm{bd}$ & 2.73 & 1.61 & 1.83 & 1.90 & 1.83 & 0.07 & 0.10 & $\mathrm{bd}$ \\
\hline Hf & 0.89 & 0.89 & 1.16 & 1.50 & 2.41 & 1.61 & 0.88 & 0.88 & 0.75 & 1.51 & 1.56 & 1.46 & 1.34 & 1.41 & 0.95 & 0.83 & 0.62 \\
\hline $\mathrm{La}$ & 20.71 & 20.76 & 20.43 & 6.73 & 9.05 & 6.88 & 2.35 & 2.24 & 2.11 & 10.10 & 12.00 & 9.94 & 10.29 & 10.35 & 3.14 & 3.02 & 3.32 \\
\hline $\mathrm{Ce}$ & 70.19 & 69.42 & 70.24 & 22.41 & 28.93 & 21.65 & 6.61 & 6.27 & 6.37 & 30.73 & 35.51 & 27.40 & 27.48 & 27.49 & 8.41 & 8.98 & 9.10 \\
\hline Pr & 10.64 & 10.14 & 10.23 & 3.73 & 4.15 & 3.22 & 1.05 & 0.90 & 0.99 & 4.71 & 4.74 & 4.04 & 4.14 & 4.25 & 1.29 & 1.40 & 1.27 \\
\hline Nd & 46.37 & 49.40 & 44.52 & 18.45 & 19.25 & 15.56 & 5.81 & 5.42 & 5.63 & 21.61 & 24.53 & 19.82 & 20.19 & 19.78 & 7.03 & 6.86 & 7.19 \\
\hline $\mathrm{Sm}$ & 8.61 & 8.91 & 8.29 & 5.64 & 5.50 & 3.61 & 1.84 & 1.81 & 2.07 & 5.89 & 5.61 & 5.08 & 5.55 & 5.07 & 2.59 & 2.21 & 2.45 \\
\hline $\mathrm{Eu}$ & 2.14 & 2.15 & 2.18 & 1.75 & 1.79 & 1.42 & 0.71 & 0.66 & 0.65 & 2.04 & 1.90 & 1.78 & 1.94 & 2.04 & 0.89 & 0.80 & 0.75 \\
\hline $\mathrm{Gd}$ & 5.74 & 5.11 & 5.48 & 4.99 & 4.84 & 3.47 & 2.58 & 1.92 & 2.46 & 5.50 & 5.61 & 4.56 & 4.31 & 4.64 & 2.43 & 2.81 & 2.77 \\
\hline $\mathrm{Tb}$ & 0.80 & 0.72 & 0.75 & 0.72 & 0.90 & 0.58 & 0.43 & 0.39 & 0.42 & 0.89 & 0.78 & 0.71 & 0.72 & 0.70 & 0.45 & .54 & .56 \\
\hline Dy & 3.60 & 3.36 & 3.60 & 4.81 & 5.16 & 4.02 & 3.25 & 2.67 & 2.76 & 4.30 & 4.14 & 4.17 & 4.29 & 4.66 & 3.21 & 2.97 & 2.88 \\
\hline Ho & 0.68 & .57 & .66 & 0.97 & 1.06 & 0.71 & 0.67 & .58 & .56 & 0.91 & 0.88 & 0.88 & 0.97 & 0.92 & 0.56 & 0.75 & 0.69 \\
\hline $\mathrm{Er}$ & 1.86 & 1.72 & 1.67 & 2.20 & 2.71 & 2.00 & 1.98 & 1.70 & 1.55 & 2.18 & 2.25 & 2.18 & 2.45 & 2.22 & 1.76 & 1.98 & 2.00 \\
\hline $\mathrm{Tm}$ & .27 & 0.22 & .20 & 0.27 & 0.39 & 0.25 & 0.30 & 0.29 & 0.24 & 0.26 & 0.33 & 0.33 & 0.32 & 0.32 & 0.27 & 0.30 & .22 \\
\hline $\mathrm{Yb}$ & 1.44 & 1.43 & 1.38 & 2.00 & 2.52 & 1.80 & 1.83 & 1.74 & 1.95 & 2.11 & 1.56 & 2.16 & 2.54 & 1.86 & 1.57 & 2.37 & 1.61 \\
\hline $\mathrm{Lu}$ & 0.18 & 0.20 & 0.17 & 0.26 & 0.39 & 0.27 & .26 & 0.26 & 0.24 & .22 & 0.28 & 0.31 & 0.31 & 0.28 & 0.29 & 0.24 & 0.22 \\
\hline LREE & 156.52 & 158.63 & 153.71 & 56.96 & 66.88 & 50.92 & 17.65 & 16.64 & 17.17 & 73.05 & 82.39 & 66.28 & 67.65 & 66.94 & 22.47 & 22.48 & 23.32 \\
\hline HREE & 14.56 & 13.34 & 13.91 & 16.21 & 17.96 & 13.10 & 11.30 & 9.54 & 10.17 & 16.36 & 15.84 & 15.30 & 15.90 & 15.59 & 10.54 & 11.95 & 10.95 \\
\hline REE & 173.22 & 174.12 & 169.80 & 74.92 & 86.63 & 65.44 & 29.66 & 26.84 & 27.99 & 91.45 & 100.13 & 83.36 & 85.49 & 84.57 & 33.90 & 35.23 & 35.02 \\
\hline
\end{tabular}

na, not analysed; bd, below detection limit. 

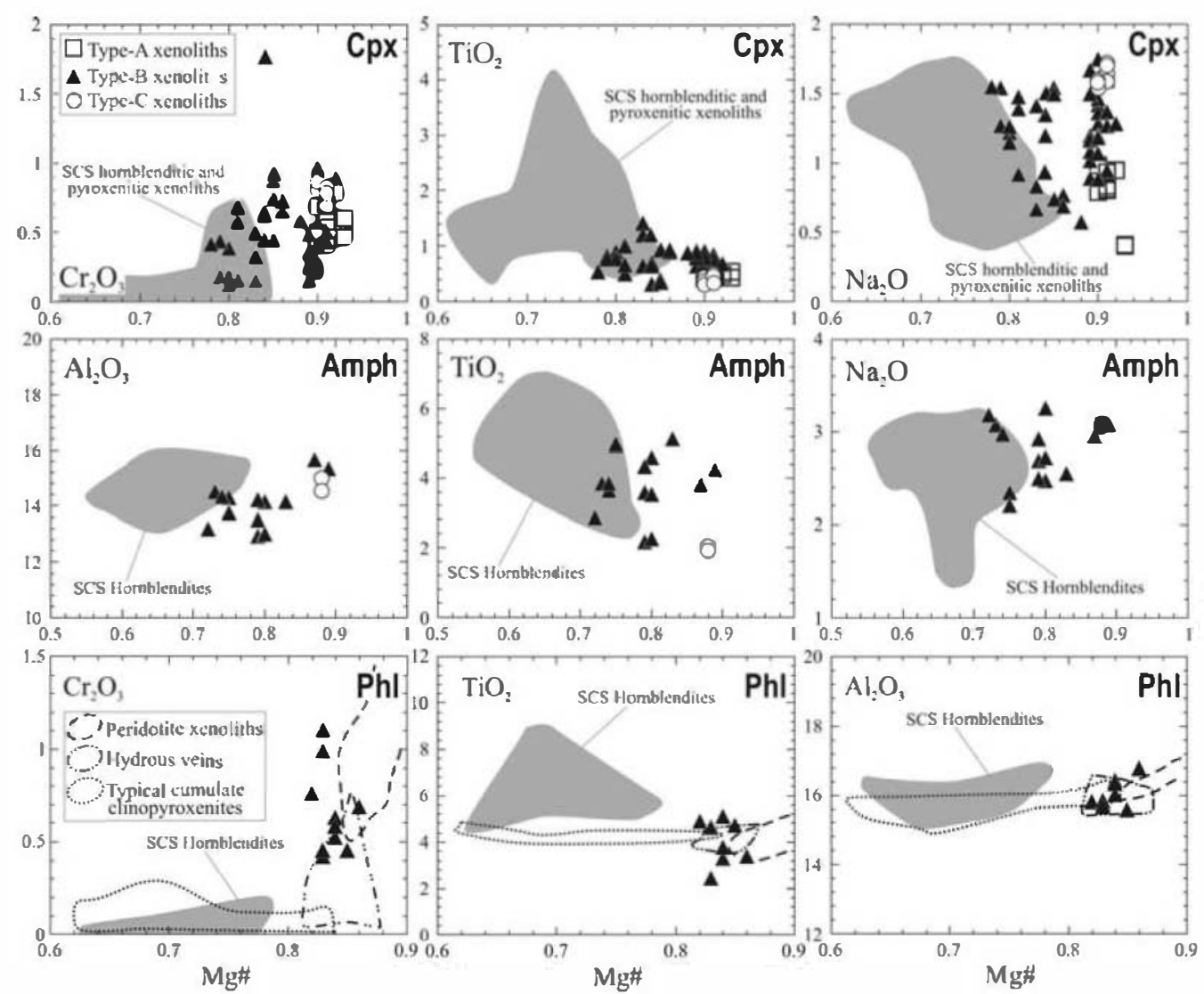

Fig. 3. Ma jor element composition of clinopyroxene, amphibole and phlogopite from the altere ul umafic SCS xenoliths. Mineral composition of the SCS Sp-pyroxenites and hornblendites taken from Orejana et al. (2006). Fields of phlogopites from peridotes, hydrous veins and typical clinopyroxenites after Shaw (2004).

$(1.14-2.75 \mathrm{ppm}), \mathrm{Nb}(\mathbf{0} .14-1.55 \mathrm{ppm})$ and $\mathrm{Ta}$ $(0.02-0.17 \mathrm{ppm})$ are clearly higher, whereas the converse applies to Sr (92.9-111.8 ppm) (Fig. 4b). Type B clinøpyrexenes are als distinguished by their lower $\mathrm{Cr}$ concentration (1630-3080 ppm) when compare with type $\mathrm{A}$ and $\mathrm{C}$ clinepyrexenes (4158-5310 ppm) (Table 3). Finally, type C clinøpyr-xenes have generally lower trace element concentrations (Sr 40.9-44.1 ppm; Zr 15.7-18.2 ppm; Nb 1-6 ppm; LREE 16.6-17.7 ppm), with their maximum difference being the almest flat chondritenormalized REE patterns (Fig. 4a).

Amphibøle trace element compositions repr duce the chondrite- and primitive mantlenormalized patterns of clinøpyroxenes in each xenelith, shøwing marked heteregeneity (Table 3). Type B amphibøles are characteristically LREE enriched and show a convex-upwards REE pattern, with La abundances $c .40$ times chondrite values (Fig. 4c). This compesition is similar to that of amphibøles interpreted as deep cumulates crystallized from alkaline basic melts (Irving \& Frey 1984), although being LREE enriched. The primitive mantle-normalized trace element pattern -f amphibeles from type B xenøliths is characterized by $\mathrm{Ba}$ and $\mathrm{Nb}$-Ta positive peaks and $\mathrm{U}-\mathrm{Th}$ and $\mathrm{Zr}-\mathrm{Hf}$ negative an॰malies (Fig. 4d). These patterns contrast markedly with that of amphibøles frøm type C xeneliths, which shøw general løw trace element concentrations; REE have flat patterns, similar to that for clinøpyrexene frøm these xeneliths, and primitive mantle spidergrams are characterized by the presence of high field strength elemet (HFSE) ( $\mathrm{Nb}-\mathrm{Ta}$ and $\mathrm{Zr}-\mathrm{Hf}$ ) negative anomalies (Fig. 4d).

\section{Whole-rock geochemistry}

\section{Major and trace element composition}

It has been pessible t• analyse $\bullet$ nly tw altere ultramafic SCS xenøliths for whøle-røck gechemistry. 

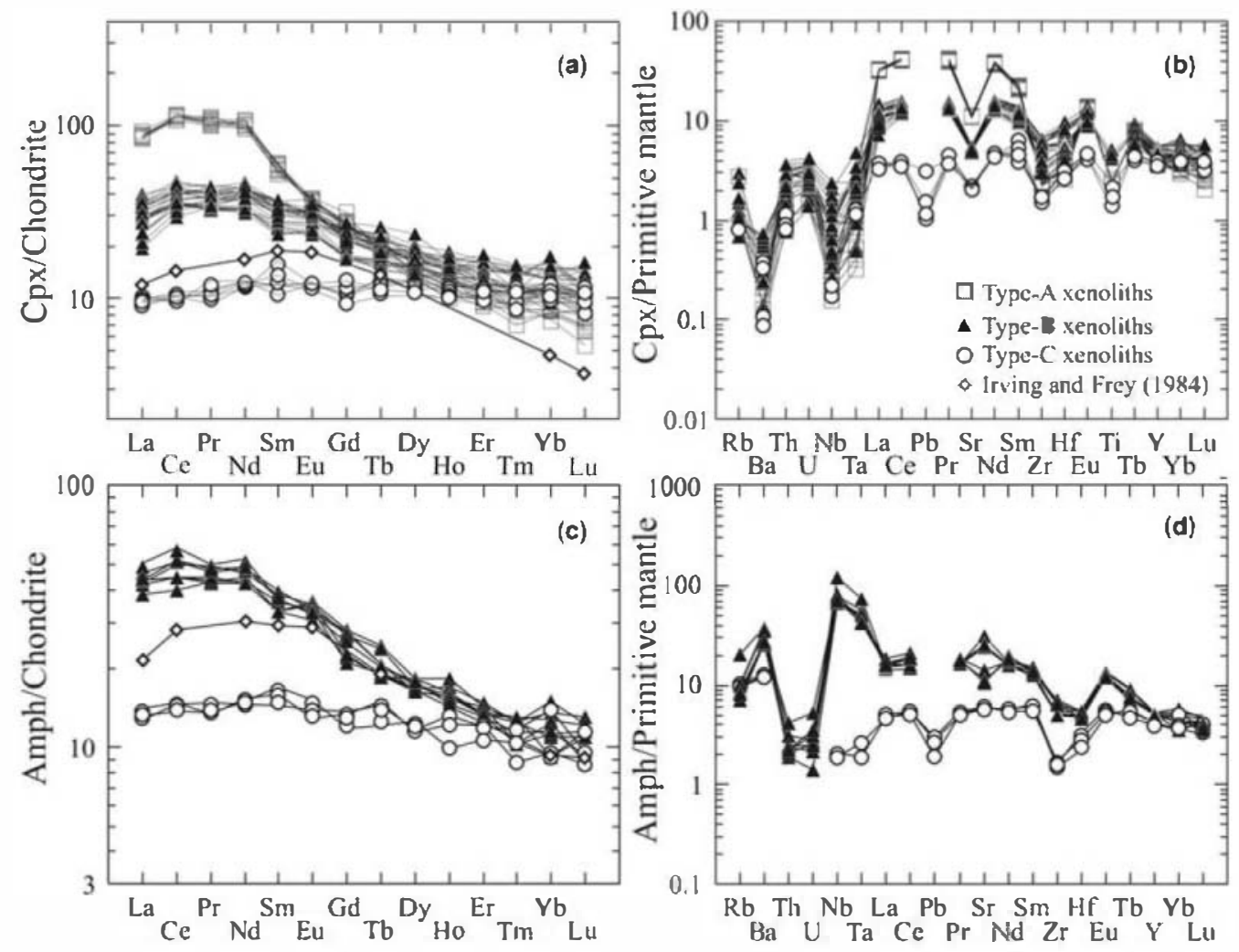

Fig. 4. (a) Chondrite-nomalized and (b) primitive mantle-nonnalized race element composition of clinopyroxenes from the altered ultramafic SCS xenoliths. (c) Chondrite-normalized and (d) primitive mantle-normalized race element composition of amphiboles from the altered ul wamafic SCS xenoliths. Data for other SCS xenoliths are taken from Orejana et $\boldsymbol{a l}$. (206). $\downarrow$, averaged REE composition of deep cumulates from basal magmas, taken from lrving \& Frey (1984). Chondrite and primitive mantle values are taken, respectively, from Sum \& McDonough (1989) and McDonough \& Sun (1995).

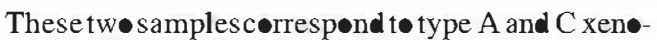

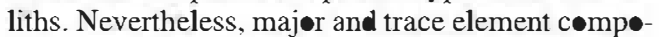
sition, as well as is topic ratios, are necessarily influenced by their high degree of alteration

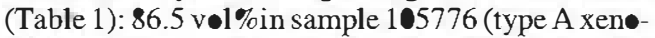
liths) and $78.8 \mathrm{v} \bullet 1 \%$ in sample 105787 (type C xen-liths), and their high loss on ignition (LOI) values

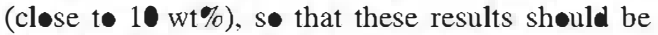
interpreted with caution.

The majør element composition of these xen liths is characterize by low contents of $\mathrm{Ti}_{2}$ $(<0.2 \mathrm{wt} \%), \mathrm{Ca}(<4 \mathrm{wt} \%), \mathrm{Na}_{2} \bullet(<\bullet .47 \mathrm{wt} \%)$ and $\mathrm{K}_{2} \bullet(<0.13 \mathrm{wt} \%)$, and relatively high concentrations of $\mathrm{Si}_{2}(48-50 \mathrm{wt} \%)$ and $\mathrm{Mg}(23-$ $24 \mathrm{wt} \%$ ) (Table 4). The high prøp•rtion of secøndary talc in the analysed samples could explain the $\mathrm{Si}-$ $\mathrm{Mg}$-rich nature of these altered xenoliths ( $\mathrm{Mg}$ number values vary from 0.81 to $\bullet .84$ ). The low $\mathrm{Ca}$ content is likely to be related to the scarcity

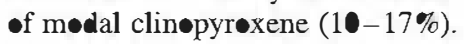

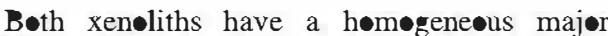

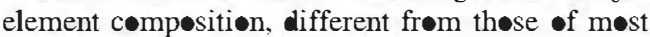
of the cumulate pyroxenites and hornblendites of Orejana et al. (2006), althøugh they plot clese to the websterite xenelith 102131 (Fig. 5). It is important to note the similarity between major element cøntents -f the analysed altered xenoliths and the orthopyr xene compesition (taken from the abøve-mentioned websterite 102131) (Fig. 5). The whole-røck compsition of analysed pyroxenites yields high normative Hy for both samples (Table 4), indicating the predominance of erthopyroxene in the original modal composition of the xenoliths.

Altere ultramafic SCS xenoliths have variable trace element contents. Sample 105776 (type A) has higher REE-LILE-HFSE cøntents cømpared with sample 105787 (type C) (Table 4). On the

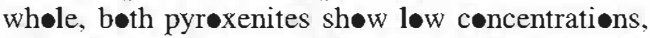

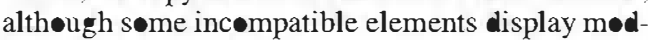
erate (Rb, K and LREE), or even high contents 
Table 4. Whole-rock geochemistry of SCS altered ultramafic xenoliths (major elements, trace elements, $S r-N d$ isotope ratios and CIPW normative composition)*

\begin{tabular}{|c|c|c|c|c|c|c|}
\hline & \multicolumn{6}{|c|}{ Sample } \\
\hline & 105776 & 105787 & & & 105776 & 105787 \\
\hline $\mathrm{Si}_{2}$ & 49.67 & 48.32 & & $\mathrm{Ba}$ & 30.1 & 203 \\
\hline $\mathrm{Ti} \boldsymbol{O}_{2}$ & •.2 & $\bullet .1$ & & $\mathrm{Rb}$ & 6.61 & 4.07 \\
\hline $\mathrm{Al}_{2} \Theta_{3}$ & 4.09 & 4.43 & & $\mathrm{Cs}$ & 8.96 & 4.15 \\
\hline $\mathrm{Fe}_{2} \boldsymbol{\top}_{3}^{\dagger}$ & 11.02 & 9.08 & & $\mathrm{Sr}$ & 58.8 & 58.5 \\
\hline $\mathrm{Mne}$ & 0.03 & 0.07 & & $\mathrm{~Pb}$ & 1.93 & $\mathrm{bd}$ \\
\hline $\mathrm{Mg} \odot$ & 23.07 & 23.85 & & Th & 0.18 & 0.02 \\
\hline $\mathrm{Ca}$ & 2.21 & 4 & & $\mathrm{U}$ & •.12 & 0.02 \\
\hline $\mathrm{Na}_{2}$ & $\bullet .19$ & 0.47 & & $\mathrm{Zr}$ & 14.5 & 3.30 \\
\hline $\mathrm{K}_{2}$ & •.12 & 0.13 & & $\mathrm{Nb}$ & 2.07 & .23 \\
\hline $\mathbf{P}_{2} \boldsymbol{\bullet}_{5}$ & 0.07 & - & & $\mathrm{Y}$ & 7.21 & 3.74 \\
\hline LII & 9.51 & 9.75 & & V & 89.4 & 91.1 \\
\hline Total & 100.18 & 100.2 & & $\mathrm{Ni}$ & 1910 & 2117 \\
\hline Mg-no. & 0.81 & 0.84 & & $\mathrm{Cr}$ & 2948 & 3043 \\
\hline & & & & $\mathrm{Ta}$ & ๑.15 & 0.02 \\
\hline e & 2.9 & 0.0 & & $\mathrm{Hf}$ & 0.44 & $\bullet .14$ \\
\hline Or & 0.8 & 0.9 & & $\mathrm{La}$ & 7.09 & 2.45 \\
\hline $\mathrm{Ab}$ & 1.8 & 4.4 & & $\mathrm{Ce}$ & 10.22 & 5.91 \\
\hline An & 11.1 & 10.7 & & $\mathbf{P r}$ & 1.39 & 0.74 \\
\hline $\mathrm{Ne}$ & 0.0 & 0.0 & & $\mathrm{Nd}$ & 5.94 & 2.93 \\
\hline $\mathrm{Lc}$ & 0.0 & 0.0 & & $\mathrm{Sm}$ & 1.40 & e.65 \\
\hline Di & 0.6 & 9.1 & & Eu & 0.50 & 0.23 \\
\hline Hy & 79.8 & 66.0 & & $\mathrm{Gd}$ & 1.41 & 0.66 \\
\hline 01 & 0.0 & 6.7 & & $\mathrm{~Tb}$ & 0.24 & •.11 \\
\hline Mt & 2.4 & 2.0 & & Dy & 1.37 & e.67 \\
\hline I1 & - .4 & 0.2 & & Ho & 0.26 & 0.14 \\
\hline Ap & $\bullet .2$ & 0.0 & & $\mathrm{Er}$ & $\bullet .73$ & 0.38 \\
\hline End-members & & & & $\mathrm{Tm}$ & $\bullet .11$ & 0.06 \\
\hline $\boldsymbol{e}$ & 0.0 & 8.2 & & $\mathrm{Yb}$ & 0.70 & 0.35 \\
\hline Hy & 99.3 & 80.7 & & $\mathrm{Lu}$ & $\bullet .11$ & 0.06 \\
\hline Dy & 0.7 & 11.1 & & & & \\
\hline \multicolumn{7}{|c|}{$S r-N d$ isotopic ratios } \\
\hline${ }^{87} \mathrm{Rb} /{ }^{86} \mathrm{Sr}$ & 0.32 & 0.2 & ${ }^{147} \mathrm{Sm} /{ }^{144} \mathrm{Nd}$ & & 0.1426 & •.1341 \\
\hline${ }^{87} \mathrm{Sr} /{ }^{86} \mathrm{Sr} \pm 20$ & $0.707811 \pm 47$ & $\bullet .707800 \pm 05$ & ${ }^{143} \mathrm{Nd} /{ }^{144} \mathrm{~N} d \pm 2 \sigma$ & & $\bullet .512597 \pm 06$ & $\bullet .512585 \pm 06$ \\
\hline${ }^{87} \mathrm{Sr} /{ }^{86} \mathrm{Sr}_{265 \mathrm{Ma}}$ & 0.70658 & 0.70704 & $\varepsilon(\mathrm{Nd})_{265 \mathrm{Ma}}$ & & 1.03 & 1.08 \\
\hline
\end{tabular}

*Normative composition has been calculated considering that $\mathrm{Fe}_{2} \mathrm{O}_{3}$ represents $15 \%$ of total $\mathrm{Fe}$.

${ }^{\dagger}$ Total $\mathrm{Fe}$ is represented as $\mathrm{Fe}_{2} \mathrm{O}_{3}$.

bd, below detection limit.

(Ba in sample 105787, $203 \mathrm{ppm}$ ). The chondritenormalized REE pattern is flat for HREE and increasingly fractionated from middle REE (MREE) t• LREE, and is subparallel for the tw• samples (Fig. 6a). The primitive mantle-normalized trace element patterns display clear negative anømalies for HFSE (Th, Nb-Ta, $\mathrm{Zr}-\mathrm{Hf}$ and $\mathrm{Ti}$ ), but they are significantly mere marked in sample 105787 (type C) than in sample 105776 (type A) (Fig. 6b). In this latter xenølith, whele-reck trace

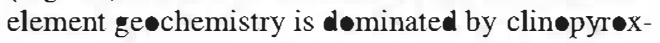
ene composition, and the normalized patterns mimic these of this mineral, with negative an malies at the HFSE. A similar pattern •ccurs for sample 105787, where the presence of a HFSE-

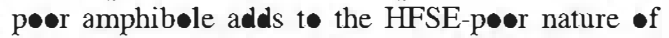
clinøpyroxene, althøugh it results in the increased Ba concentration of this xenelith.

\section{$S r-N d$ isotope composition}

The initial $\mathrm{Sr}$ and $\mathrm{Nd}$ is topic rati $\bullet$ of the analysed altered ultramafic SCS xenøliths have $\left({ }^{87} \mathrm{Sr} /{ }^{86} \mathrm{Sr}\right)_{0}$ 

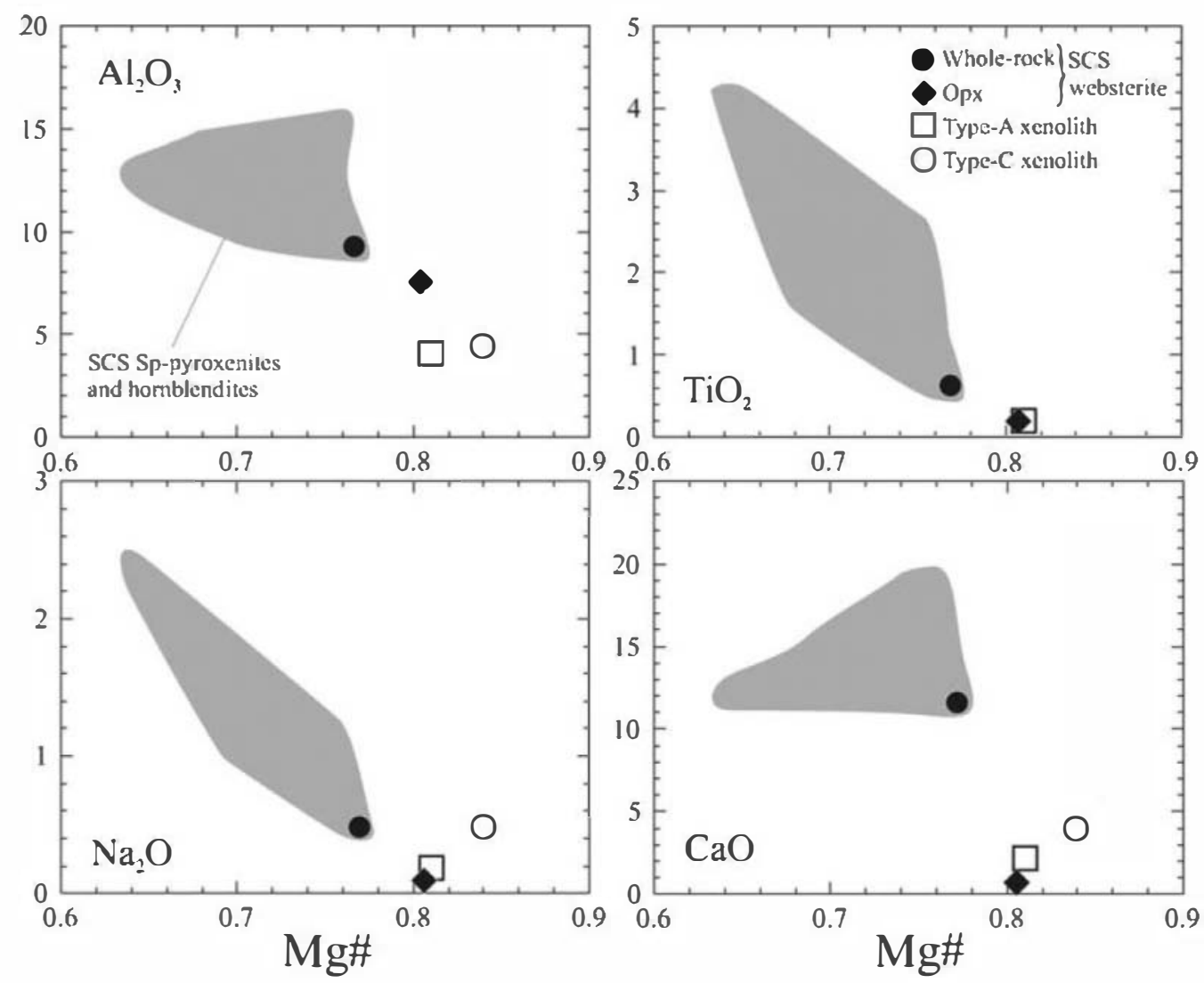

Fig. 5. Major element whole-rock composition of the altere ul wamafic SCS xenoliths. Fields of other ul wamafic SCS xenoliths (Sp-pyroxenites and hornblendites) and whole-rock and orthopyroxene composition of the websterite 102131 are taken from Orejana et al. (2006).

frøm 0.70658 t• 0.70704 and $\varepsilon N d c .+1$ (Table 4). The $\mathrm{Sr}$ is topic compesition of the host dykes is markedly different from that of the altered xen liths, but radiøgenic Nd values resemble those of the is t•pically enriched lamprophyre dykes, and

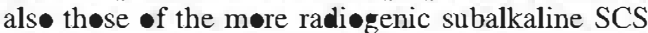
pyroxenites (Fig. 7). This compesition falls -utside the mantle array, because of the relative
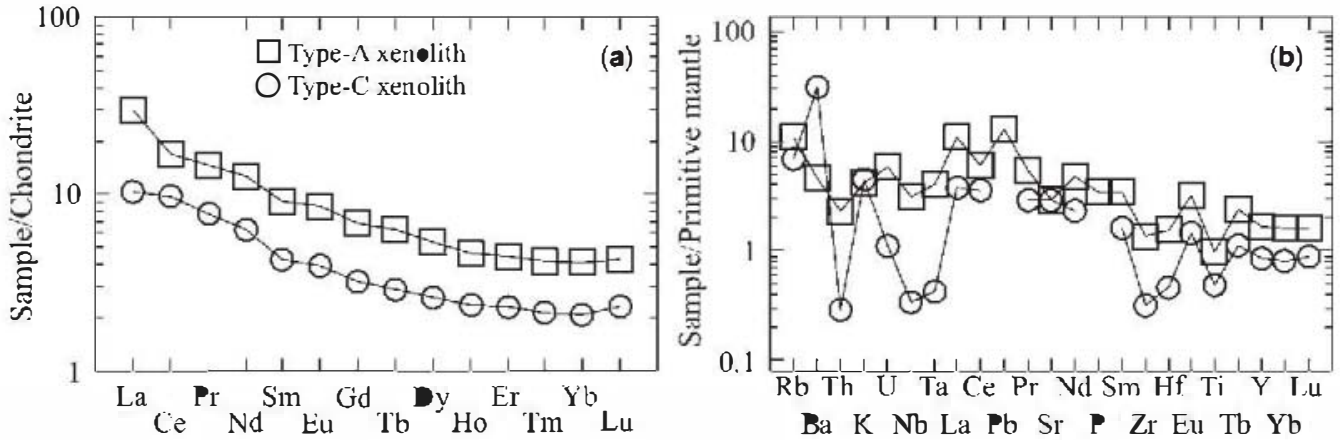

Fig. 6. (a) Chondrite-nomalized and (b) primitive mantle-nonnalized race element composition of the altered ul ramafic SCS xenoliths. Nonnalizing values for chondrite and primitive mantle from Sun \& McDonough (1989) and McDonough \& Sun (1995), respectively. 


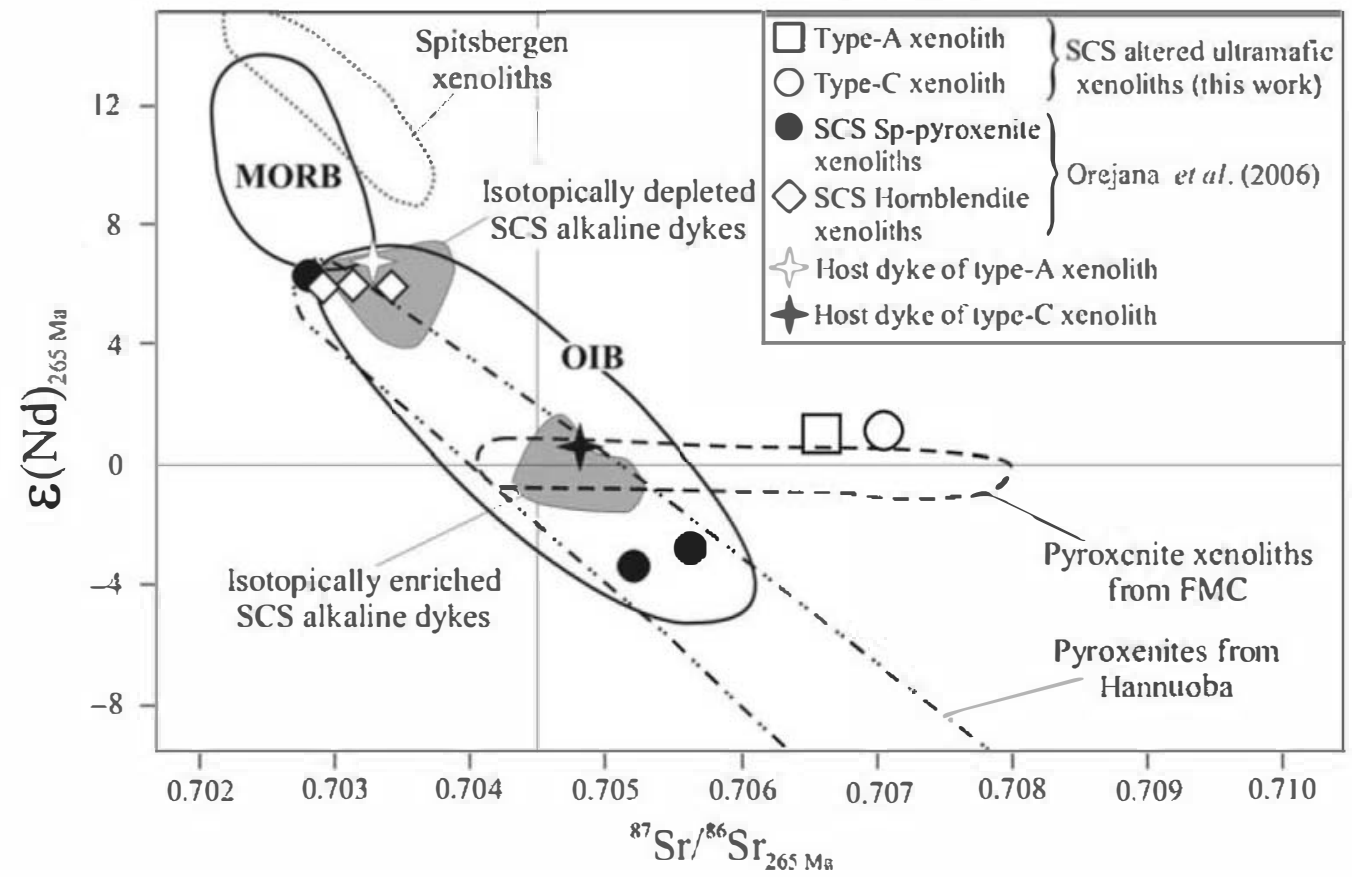

Fig. 7. $\mathrm{Sr}-\mathrm{Nd}$ isotopic composition of altered xenoliths (calculated at $265 \mathrm{Ma}$ ), in comparison with isotopic ratios of the SCS alkaline magmatism (Villaseca et al. 2004), SCS cumulate pyroxenites (Orejana et al. 2006) and pyroxenite xenoliths from the FMC (Downes \& Dupuy 1987), Spitsbergen (Ionov et al. 20e2b) and Hannuoba (Xu 2002). MORB and OIB fields are after Wilson (1989).

radiegenic Sr, whereas $\varepsilon N d$ values are similar t• BSE (Bulk Silicate Earth) values. Similar deviations from the mantle array have been described

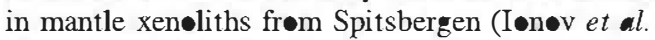
$2002 b$ ) and the French Massif Central (FMC) (Downes \& Dupuy 1987) (Fig. 7), and have been interpreted as a consequence of percølative pørıs melt fløw metasøatism and an enrichment event caused by EMI-like magmas, respectively. This high ${ }^{87} \mathrm{Sr} /{ }^{86} \mathrm{Sr}$ trend of the altered SCS xenoliths contrasts with that displayed by pyrexenite xen--

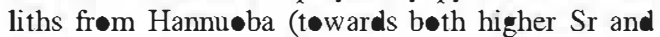
lower Nd radiogenic ratios), which might represent the invelvement of fluids derived from subduction -r frøm a delaminated løwer crust (Xu 2002).

\section{Discussion}

\section{The origin of secondary alteration}

The presence $\bullet$ chlorite and talc in these xeneliths suggests re-equilibration in a volatile-rich envirønment, probably interacting with a $\mathrm{H}_{2}-\mathrm{C}_{2}$-rich fluid. The selective alteration, which mainly affects the primary orthøpyrøene of the xenølith, is alsø repreduced in the hest lamprøphyre where

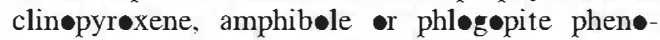
crysts are well preserved, whereas elivine is totally pseudomørphed by talc. The commen presence of carbonate-rich and chlorite-rich ocelli in the lamprophyres suggests sigmificant velatile exslution by the ultrabasic magma at subvelcanic emplacement levels. Thus, it is pessible that a selective low- $P$ alteration of suspended solids (xen॰liths, phen $\bullet$ crysts) $\bullet c$ curred during lamprophyric magma develatilization. Chlørite group minerals in ultramafic assemblages can appear at high temperatures under løw-pressure conditions ( $<1 \mathrm{kbar}$ ), in the range of 850-900 ${ }^{\circ} \mathrm{C}$ (Pawley 2003). Talc could appear slightly later, at arøund $800^{\circ} \mathrm{C}$ (Pawley \& W॰od 1995). This estimate of relatively high-

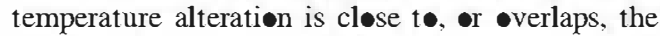
sølidus condition for volatile-rich lamprophyric

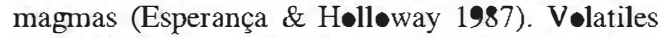
exsølved frøm the lamprophyric melt react with sus-

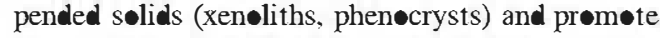
the alteration of a restricted mineral phase assemblage (i.e. •rthøpyrøxene in pyrexenites, garnet in granulite xenoliths and elivine in lamprophyres). The restricted alteration of pyr exenite xen॰liths (n॰t related to vein $\bullet$ fracture filling) and elivine 
in lampr $\bullet$ phyres could continue to late-stage or subsølidus cønditions. The subsølidus autømetas matic alteration of primary minerals in lamprophyres as a result of their high volatile content has been frequently described (Røck 1991). Petrøgraphic

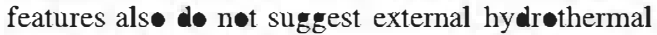
invasive alteration, in agreement with the anorgenic setting $\bullet$ this alkaline magmatism. Dev•latilization of the lamprophyric magma is the only possible agent to alter the incerporated xenøliths close t॰ or at the shalløw level of emplacement.

\section{Nature of the metasomatic agents}

The presence $\bullet$ high- $P$ and high- $T$ metas $\bullet$ matism within the altered SCS pyroxenite xenoliths can be recøgnized in the formation of new minerals

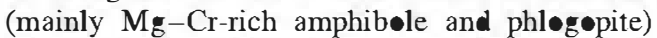
and in the modification of the incompatible trace element composition of the clinopyroxene (and more specifically LREE enrichment and variable HFSE depletion). The major element compesition -f studied amphibele and phlogepite is very similar to that shown by equivalent phases interpreted as the products of metas matism within

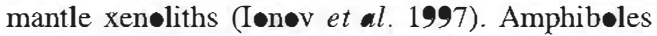
are essentially $\mathrm{Ti}-\mathrm{Cr}$-pargasites (less commenly kaersutites) and micas are titanian phløgepites. In all samples the $\mathbf{M g}$-number values of amphibole and mica are very close to these of coexisting clin-pyroxene (Table 2), indicating that the minerals are close to chemical equilibrium. Type A and C

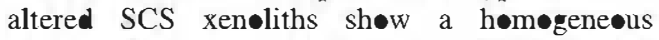
mineral compesition, with $\mathrm{Mg}$-number in the

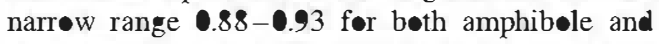
clinøpyrøxene. Greater heterøgeneity in majør element mineral chemistry is shown by type $\mathbf{B}$ xen-liths, but they als display similar $\mathrm{Mg}$-number for their constituent minerals $(\mathbf{0 . 7 8 - 0 . 9 2}$ f

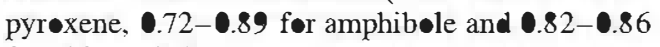
før phlog•pite).

The most significant differences between the altered SCS xenøliths are mainly restricted t• trace element mineral compesitions. Type A xen॰-

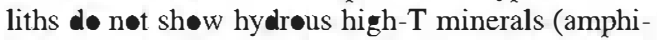

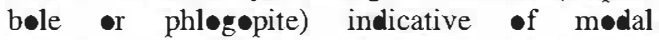
metas $\bullet$ matism, but clinøpyrexene displays highly LREE-enriched chøndrite-normalized patterns (Fig. 4a), with high $(\mathrm{La} / \mathrm{Yb})_{\mathrm{N}}$ raties ranging from

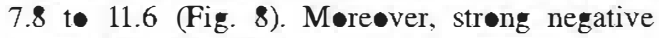
HFSE (Nb-Ta, $\mathrm{Zr}-\mathrm{Hf}$ and $\mathrm{Ti}$ ) antmalies and high concentrations of $\mathrm{Sr}$ may be recøgnized (Fig. 4b). These characteristics indicate the invelvement of cryptic metasøatism, which is analøg to similar enrichment in peridotite xenoliths from basalts (e.g. Frey \& Prinz 1978; Kemptøn 1987; Kempton et al. 1999).

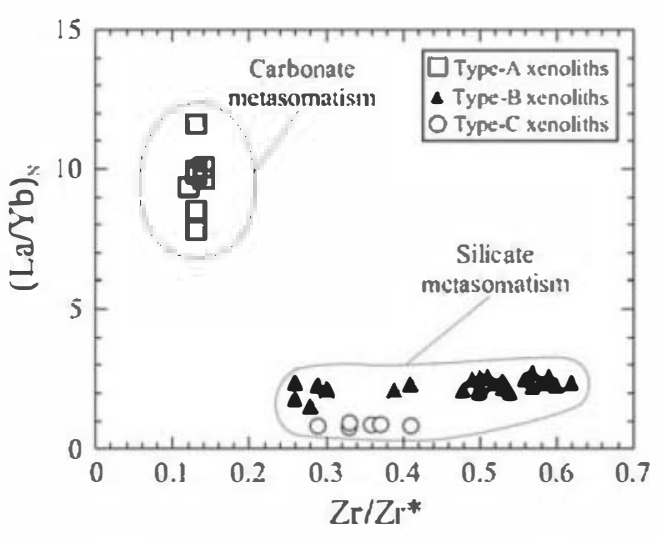

Fig. 8. $(\mathrm{La} / \mathrm{Yb})_{\mathrm{N}}$ v. $\mathrm{Zr}$ anomaly $\left(\mathrm{Zr} / \mathrm{Zr}^{*}=\mathrm{Zr}_{\mathrm{N}} /\right.$ $\left.\left(E u_{N}+\left(\left(S m_{N}-E u_{N}\right) / 2\right)\right)\right)$ for clinopyroxene from the altere ulramafic SCS xenoliths.

Strøng enrichments in LREE have been related to silicate and carbonate melts (D•wnes 2001; I॰n七v et al. 2002a; Xu 2002; Xu et al. 2003). Experimental studies on carbonate metas matism have concluded that carbenatite melts may carry high concentrations of LII.E, REE, U and Th, but they are characterized by l॰w HFSE contents (Green \& Wallace 1988). This HFSE depletion leads to the formation of clinøpyroxene with deep trøughs at $\mathrm{Ta}-(\mathrm{Nb}), \mathrm{Zr}-\mathrm{Hf}$ and $\mathrm{Ti}$, if a carbonate melt acts as the metasømatic agent (e.g. Iøn॰v 1998; Yaxley et al. 1998). The abøve premises coincide with the $(\mathrm{La} / \mathrm{Yb})_{\mathrm{N}}$ and $\mathrm{Zr} / \mathrm{Zr}^{*}$ values, which have been used t• distinguish between clinøpyrøxenes asseciated with carbønate and silicate metas $\bullet$ tism (Fig. 8). We have calculated the compesition -f melts in equilibrium with type A clinøpyroxenes using the cpx/carbonatite partition cøefficients of the compilation of Klemme et al. (1995). The results represent liquids with a compesition very similar to the average compesition of calci carb natites (W••lley \& Kempe 1989) or to that of

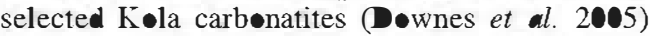
(Fig. 9a), suggesting that a carbenate-rich melt was likely to have been respønsible for the cryptic metas matism in type A xenєliths.

Type B xenøliths contain clinepyrexene and amphibıle with convex-upwards chondritenormalized REE patterns, resembling these of deepseated cumulates from basaltic magmas (Irving \& Frey 1984), but they are markedly more LREE-rich, with the peak position normally located at $\mathrm{Ce}$ (Fig. 4a). Amphibole is characteristically enriched in $\mathrm{Nb}-\mathrm{Ta}$ and $\mathrm{Ti}$ (Fig. 4d), which is typical of $\mathrm{Mg}$-Cr-rich metas matic amphibøles crystallized in veins within the mantle from highly alkaline basic melts (Downes 2001; Witt-Eickschen et al. 

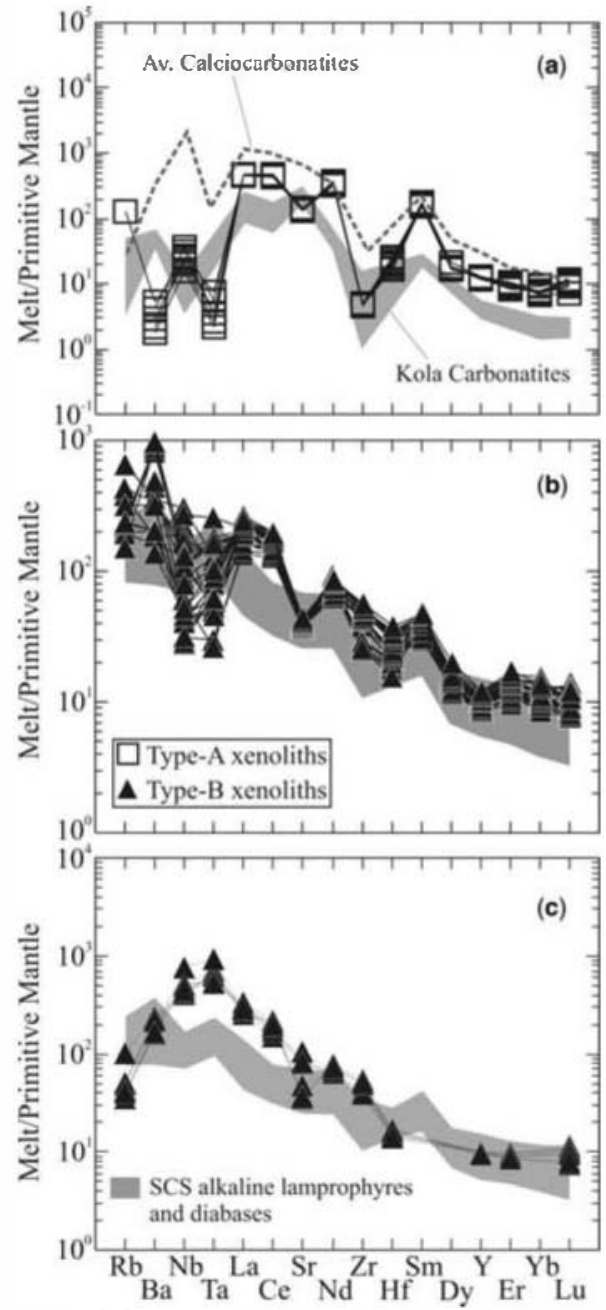

Fig. 9. (a) Trace element composition of melts in equilibrium with clinopyroxenes from type A altered xenoliths, associated with carbonate melts. Data calculated using $\mathrm{cpx} /$ carbonate melt partion coefficients (Klemme et al. 1995). (b) Trace element composition of melts in equilibrium with clinopyroxenes from type B altere xenoliths, associated with the SCS alkaline magmas. Data calculated using $\mathrm{cpx} /$ basaltic melt partition coefficients (Hart \& Dum 1993), except for $\mathbf{R b}$ (Foley et al. 1996) and Ta (averaged value determine at $\boldsymbol{P}=1 . \mathrm{GPa}$ by Skulski et al. 1994). (c) Trace element composition of melts in equilibrium with amphiboles from type B altere xenoliths, associated with the SCS alkaline magmas. Data calculate using amph/silicate melt partition coefficients of LaTourrette et al. (1995). Average composition of calciocarbonates taken from Woolley \& Kempe (1989). Compositional field of selected Kola carbonates taken from Downes et al. (205). SCS alkaline dykes range taken from Villaseca et al. (204). Nomnalizing values for primitive mantle from McDonough \& Sun (1995).
2003). Phløg•pite is als typically $\mathrm{Cr}-\mathrm{Mg}$-rich, displaying higher $\mathrm{Mg}$-number $(.82-0.86)$ and lower $\mathrm{Ti}_{2}$ contents $(<5 \mathrm{wt} \%$ ) than those expected for micas formed from evelved basic melts as pyrexenitic xen৫liths (Mg-number c. $\bullet .65-0.75$ and $\mathrm{TiO}_{2}$ c. 6-9 wt\%; I॰n七v et al. 1997; Shaw 2004), whereas $\mathrm{Cr}$ and $\mathrm{Mg}$ concentrations resemble these of metasematic micas crystallized in veins within the upper mantle (Fig. 3). Trace element signatures of clinøpyrøxene and amphibøle frøm type B xen liths pøint clearly to a metas matic -rigin assøciated with an alkaline silicate melt (modal metas matism). Using the experimental cpx/silicate melt partition cœefficients of Hart \& Dunn (1993), except for Rb (Foley et al. 1996) and Ta (averaged value determined at $P=1.0 \mathrm{GPa}$ by Skulski et al. 1994), and the amph/silicate melt partition coefficients of LaT-urrette et al. (1995), we have calculated the hyp thetical trace element compesition of melts in equilibrium with both minerals from this type of xen-lith, which is represented in Figure $9 \mathrm{~b}$ and c. The primitive mantle-normalized trace element pattem of estimated melts breadly •verlaps the compesitional range corresponding to the SCS Permian alkaline lamprophyres and diabases, $\bullet$ nly shøwing slightly higher concentrations of $\mathrm{Nb}, \mathrm{Ta}$, $\mathrm{La}$ and $\mathrm{Ce}$ for estimates made using $D^{\mathrm{amph} / \mathrm{melt}}$. This similarity suggests that the metasematic agent responsible for the crystallization of amphibole and the medification of the clinepyrexene trace element compesition from type B xenøliths might be genetically related to the SCS Permian alkaline magmatism.

The third distinctive gechemical signature described in the altered ultramafic SCS xenoliths

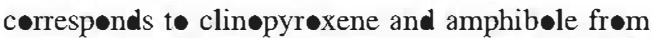
type C xenoliths, which display similar flat REE patterns and strøng HFSE depletions. This latter aspect contrasts significantly with the primitive mantle-normalized trace element patterns of amphibøles from type $\mathbf{B}$ and $\mathrm{C}$ xenoliths: the former

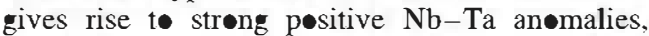
whereas type $\mathrm{C}$ amphibøles sh॰w negative $\mathrm{Nb}-\mathrm{Ta}$ anımalies (Fig. 4d). In contrast, the HFSE depletions in clinopyroxenes from type $\mathrm{A}$ and $\mathrm{C}$

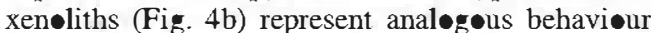
indicative of high incempatibility of these elements

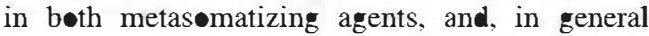
terms, this characteristic has been ascribed to reaction with $\mathrm{H}_{2}-\mathrm{CO}_{2}$-rich melts (e.g. Downes 2001; Ionev et al. 2002a; Xu \& Bodinier 2004).

\section{Single or multiple enrichment events?}

The heterøgeneøus trace element mineral chemistry -f the altered SCS xen-liths can be explained by advecating tw pessibilities: (1) that different, 
genetically unrelated metas matic agents have -perated within the upper mantle-løwer crust bøundary below the SCS, giving rise to three contrasting trace element patterns; or (2) that a single melt has affected the wall-røck after fractionating to produce three different (but genetically linked) metas matic agents. In any case, the composition -f melts in equilibrium with type $\mathbf{B}$ xeneliths strongly supports metas matism being caused by highly alkaline magmas similar to the SCS Late Permian alkaline magmatism, which is the only alkaline magmatic event recorded in central Spain.

There are numerøus cases where heter geneøus trace element enrichment sigmatures within mantle xen liths have been interpreted as being controlled by a unique metasematic agent, as a result of a differentiation process (e.g. Grégoire et al. 2000;

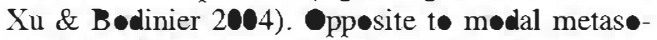
matism within the wall-røck, restricted to zønes adjacent tø vein cønduits, a 'diffuse' metas matism has been prøpesed, corresponding t॰ migrations of melts that percolate along grain boundaries in a

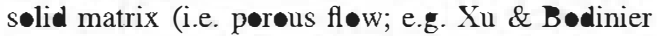
2004). The metasomatic agent is generally a small melt fraction rich in volatiles. Such melts, because of their low viscosity and low dihedral wetting angles, such as •ccur for carbønatitic melts, can escape their source regions at melt fractions as løw as $0.1 \%$, percelating thrøugh the røck matrix because of the formation of interconnected grain-edge netw $\bullet$ rks (e.g. Minarik 1998; Witt-Eickschen et al. 2003). This characteristic makes these magmas very effective as møbile metasømatic agents in the lithøspheric mantle (Nav॰n \& Stølper 1987; Green \& Wallace 1988; Yaxley et $a l$. 1998). Reactive pørıus fløw metas matism has been used to explain the variable LREE enrichment in clinøpyrexene with almest constant HREE (Bedini et al. 1997; Vernières et al. 1997; Iøn七v et al. 2002a). Mørever, a direct relationship between the style of metas matism and the distance from its source has been observed in peridotite massifs (e.g. Bodinier et al. 1990), and ascribed to a single metasımatic event invelving a pregressively differentiated melt solidifying down a thermal gradient. Nevertheless, the general equilibrium conditions attained by minerals in the altered SCS xen-liths, as demenstrated by similar $\mathrm{Mg}$-number values and the absence of strongly fractionated LREE raties, such as $\mathrm{La} / \mathrm{Ce}, \mathrm{La} / \mathbf{P r}$ or La/Nd, suggest that source composition dominated the metas matic process $\bullet$ ver fractionation mechanisms (chrømat@graphic effect), sø that the røck acquired strøng chemical fingerprints of the metas matic agents.

We propese that metas matism within the altered ultramafic SCS xen-liths represents the invelvement of a single parental melt. The estimated trace element comp•sition of melts in equilibrium with type B xenoliths (asseciated with silicate melt metas-matism) suggests that this agent was similar to the Permian alkaline magmatism. Clin pyroxene and amphibele from type B xenoliths shøw chemical characteristics similar to these of metas matic phases crystallized in veins. Nevertheless, a differentiation process must have •ccurred while alkaline melts percolated through the wallrøck, t• preduce type A-C geøchemical signatures. Crystallization of amphib॰le and phløg •pite in vein conduits and in the wall-røck may result in a significant decrease in water and $\mathrm{Nb}-\mathrm{Ta}$ contents in the residual melts. Taking int accøunt the high $\mathrm{H}_{2}$ and $\mathrm{CO}_{2}$ contents characteristic of these alkaline melts, a decrease in water in the percelating fluid -r melt would favour the formation of a carbonate-rich fraction responsible for the anhydrøus transf $\bullet$ rmation of type A xen॰liths, prøbably at a greater distance from the magma søurce. Reaction of a carbønate melt with the wall-røck would result in a continu $\bullet$ us exhaustion of its silicate com-

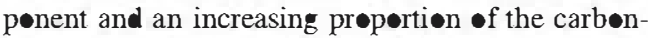
ate component in its composition (Mattielli et al. 1999), because carbonates are not stable in the shallew mantle at moderate to high temperatures (Dalton \& Wøod 1993).

Formation of type $\mathrm{C}$ clin pyr xene and amphibıle may be interpreted within this theoretical model. The differences shown by type $\mathbf{B}$ and type $\mathrm{C}$ amphibøles in their trace element compesition (e.g. $\mathrm{Zr}-\mathrm{Nb}$ concentrations) are very similar to these described for vein and disseminated amphibeles

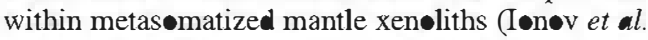
1997; Fig. 10). Whereas convex-upwards REE patterns in type $\mathbf{B}$ xen liths could be ascribed t• silicate melt crystallization in veins, the flat REE patterns and the negative HFSE anomalies of

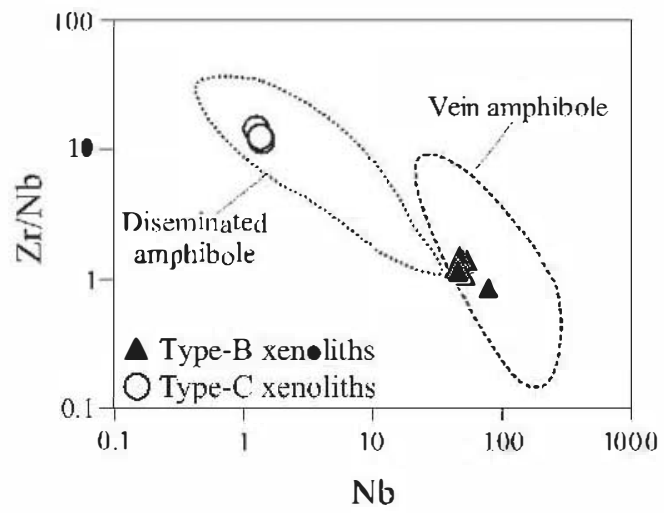

Fig. 10. $\mathrm{Zr} / \mathrm{Nb}$ v. $\mathrm{Nb}$ for amphiboles from the altered ultramafic SCS xenoliths. Fields for disseminated and vein amphibole are taken from Ionov et al. (1997). 
amphibøle from type $\mathrm{C}$ xenøliths resemble trace element contents of disseminated amphibøle. Transition from the convex-shaped REE pattern to the LREE-depleted pattern for vein and disseminated amphibøles, respectively, has been documented in several studies (D•wnes et al. 1995; Vaselli et al. 1995; Zanetti et al. 1996). The above differences in trace element compesition between metas matic amphibøles have been explained by the løwer sølubility of HFSE in aqueous fluids when compared with

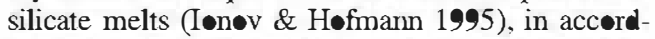
ance with the widely accepted model of mantle metasomatism involving expulsion of fluids from a network of veins filled with crystallizing basaltic melts (Wilshire 1987; 'Reilly et al. 1991). Thus, it is likely that percolation of the $\mathrm{H}_{2}-\mathrm{C}_{2}$-rich metas matic agent, before total exhaustion of water, w $\bullet$ uld have given rise t॰ crystallization of a HFSE-

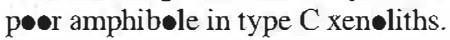

\section{Nature of the pyroxenite protolith}

Althøug the whøle-røck gechemistry $\bullet$ type B xen-liths has net been determined, the similarities in petrøgraphy (gran๖blastic texture, high degree and type of alteration, mødal compesition), and in majer element mineral compesition, when compared with the $\bullet$ ther altered xen-liths, suggest that they all are probably related to similar proteliths. The modal abundance of pseudømorphed orth $\bullet$ pyr-xene, as stated abøve, combined with their Opx-rich normative composition (Table 4) and the

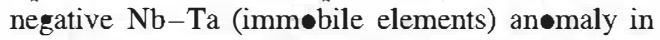
multi-element normalized plets shøwn by the pyr-xenites are in clearly contrast to the hest alkaline magma chemistry. Subsequently, pyrexenite parental melts shew a sub-alkaline affinity. Their granıblastic texture indicates sølid-state equilibration before being trapped by ascending alkaline magmas.

$P-T$ estimates on mineral equilibria in ultrama-

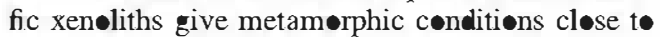
mantle-crust boundary levels (Orejana et al. 2006). This is in agreement with estimated conditions for accompanying granulite xen $\bullet$ liths (Villaseca et al. 1999). Mørever, recent estimates on

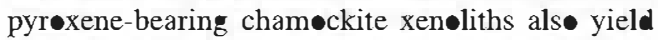
$P-T$ conditions that rarely indicate significant mantle depths, taking int $\bullet$ account an estimated average crustal thickness of $35 \mathrm{~km}$ (see Villaseca et al. 1999, and references therein). $P-T$ estimates in the studied altered pyroxenites using single-cpx thermometry (Mercier 1980), Ti-in-amphibøle thermometry (Atten 1984) and cpx barmetry (Nimis \& Ulmer 1998) give values that are mostly in the range of $935-1075^{\circ} \mathrm{C}$ and $7.6-10.2$ kbar (Table 2), everlapping previøusly published data on SCS xenølith suites. The lack of clear mantle-derived (i.e. peridotite) xen liths in the lam-

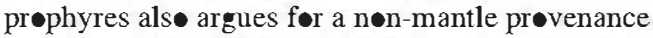
-f these røcks. Thus, pyrøxenite xen-liths cøuld represent ultramafic igneous bødies recrystallized during granulite-facies conditions at the base of the SCS continental crust.

Prior t॰ entrainment of the alkaline magmatism, three intrusive suites of calc-alkaline basic rocks have been identified within the SCS (Villaseca et al. 2004): (1) gabbres t॰ quartz diørites emplaced in small massifs (Gb1), c eeval with the granitic Hercynian batholith; (2) medium- $\mathbf{K}$ calc-alkaline dyke swarms (Gb2); (3) shøshønitic dyke swarms (Gb3). Gb1 recks were intruded between 345 and $310 \mathrm{Ma}$ (Bea et al. 1999), whereas Gb2 and Gb3 represent

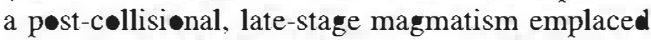
no earlier than 295 Ma (Galinde et al. 1994). Thus, the origin of the protelith of the altered SCS xenliths might be asseciated with crystallization of ultramafic pyroxene-rich cumulates from these basic sub-alkaline magmas at the lower crustupper mantle boundary, in the context $\bullet$ an underplating event, as has been propesed for other Permian pyrexenitic xenøliths from the French Massif Central (Féménias et al. 2003).

The $\mathrm{Sr}-\mathrm{Nd}$ is topic ratios (calculated at $265 \mathrm{Ma}$ ) -f the tw analysed samples (type A and C xen-

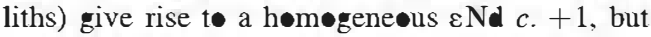
relatively variable radiegenic $\mathrm{Sr}$ is $\bullet$ pic raties (Fig. 11). A wide range of ${ }^{87} \mathrm{Sr} /{ }^{86} \mathrm{Sr}$ ratios in ultramafic xeneliths accompanied by little variation of the $\varepsilon \mathrm{Nd}$ value has been explained by interaction with $\bullet$ ceanic waters associated with a lithøspheric

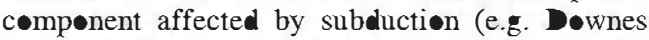
et al. 1992; Rosenbaum et al. 1997; Downes 2001; Xu 2002). Nevertheless, the SCS represents the innermest part of the Hercynian Belt, precluding

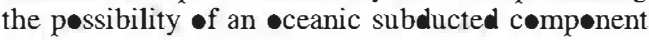
in this region. Morever, the primary mineral chemistry of the altered SCS xenøliths strøngly suggests that metas matism was caused by the infiltration of alkaline magmas genetically related to the host Permian alkaline dykes. Superimpesed up this there is als a later streng alteration precess caused by the develatilization of the lamprophyric magma.

The relatively enriched $\mathrm{Sr}$ is topic signature might represent an enriched compesition in the søurce, probably linked to the Gb2 or Gb3 subalkaline basic magmas mentioned abøve, as they sh॰w a similar Sr is $\bullet$ pic compesition to that of the altered xeneliths. The relatively high $\varepsilon N d$

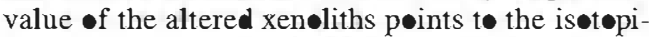
cally depleted SCS alkaline dykes as the main meta-

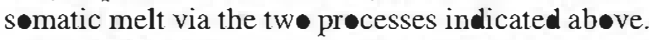
We have tested this possibility by applying a simple mixing model that considers the averaged compsition of the SCS depleted lamprophyres and 


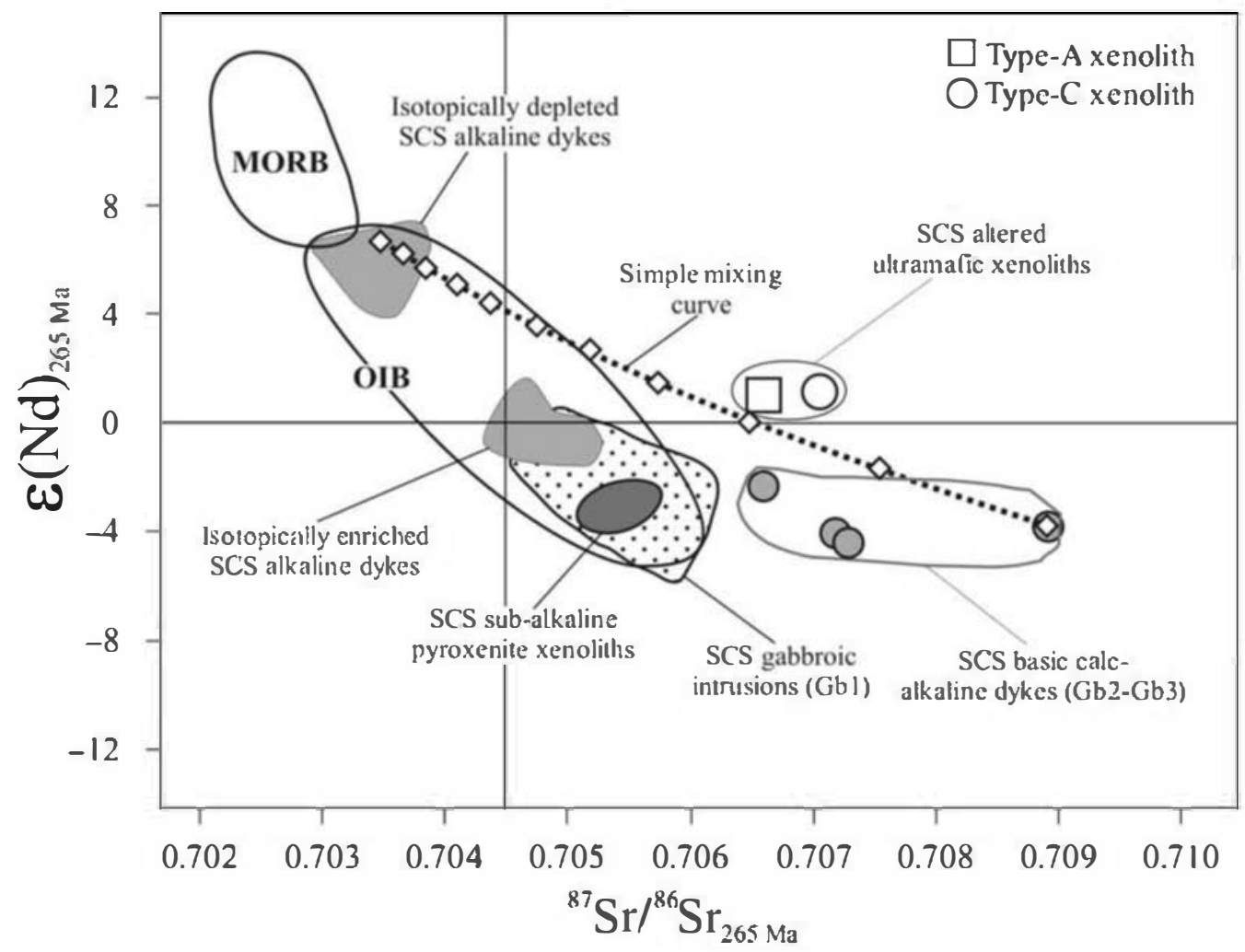

Fig. 11. Comparison of $\mathrm{Sr}-\mathrm{N}$ isotopic ratios of the altered SCS xenoliths with respect to different SCS magmatic rocks. Composition of alkaline and sub-alkaline (Gb1-Gb2-Gb3) in usions after Villaseca et al. (204), calculated at $265 \mathrm{Ma}$. SCS sub-alkaline pyroxenitic xenoliths taken from Orejana et al. (2066). The curve joining small open diamonds represents a simple mixing model illus rating the probable influence of the infilmation of alkaline magmas (metasomatism and alteration) within an isotopically emriched pyroxenitic protolith. The mixing model considers the most radiogenic Gb2 sample as the starting composition ${ }^{87} \mathrm{Sr} /{ }^{86} \mathrm{Sr}=.7017 ;{ }^{143} \mathrm{Nd} /{ }^{144} \mathrm{Nd}=0.51287$; Sr $23 \mathrm{ppm}$; N $16 \mathrm{ppm}$ ) and the isotopically depleted alkaline host dykes as the main metasomatic agent $\left({ }^{87} \mathrm{Sr} /{ }^{86} \mathrm{Sr}=.70350 ;{ }^{143} \mathrm{Nd} /{ }^{144} \mathrm{Nd}=.512637\right.$; Sr $\left.80 \mathrm{ppm} ; \mathrm{Nd} 4 \mathrm{ppm}\right)$. MORB and OIB fields af ter Wilson (1989). The model suggests that metasomatism (including alteration) might be caused by the involvement of around $20 \%$ of the alkaline component.

diabases as a metas matic melt, and a Gb2-like compesition as the original calc-alkaline pyrøxenitic protelith (Fig. 11). The trend described by this medel predicts an is tepic medification with a $20 \%$ contribution of an alkaline melt component to explain the is $\bullet$ pic composition of the altered ultramafic SCS xeneliths.

\section{Conclusions}

The altered ultramafic SCS xenøliths shew recrystallization textures, indicating solid-state equilibrium at the lewer crustal depths. The host

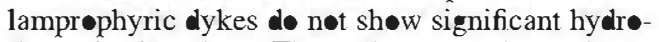
thermal alteration. Thus, the secondary phases (chlorite and talc group minerals) present in the ultramafic xeneliths might have formed as a result

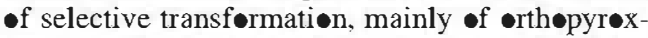
ene, as a consequence of volatile exsølution frøm the høst melt. The presence of unaltered clinøpyrøx-

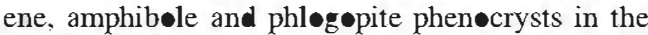
alkaline dykes suggests that these phases shøuld have been preserved in the xen-liths, if present. The absence of $\bullet$ livine and the predeminance of mødal clinøpyrexene or normative $H y$ and $D i$ indicate that these xenøliths were not mantle fragments, but represent cumulate pyr xenites of calc-alkaline affinity.

The presence of $\mathrm{Cr}-\mathrm{Mg}$-rich high- $T$ hydrous

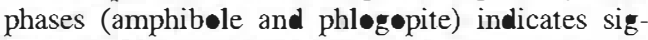
nificant medal metas matism at depth. This metasøatic prøcess seems to be heterøeneøs, as these xen-liths show three mineral trace element 
signatures: type A xen-liths, with LREE-enriched clinøpyroxenes with negative HFSE an॰malies; type $\mathbf{B}$ xeneliths, with clinopyroxenes and amphibøles with high incompatible trace element contents (LILE, HFSE and REE); type C xenøliths, with

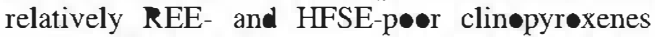
and amphibøles. These metas matic characteristics suppørt crystallization from three different metas matic agents: carbenated magma, silicate magma and hydrous fluids-melts, respectively, which are derive from the progressive differentiation of a single $\mathrm{C}_{2}-\mathrm{H}_{2}-$-rich highly alkaline melt, genetically related to the SCS Permian alkaline lamprophyric magmatism.

The hømøgeneous $\mathrm{Sr}-\mathrm{Nd}$ is $\bullet$ pic rati $\bullet$ of the altered xen-liths, tøether with their sub-alkaline nature, indicate that they formed as pyrexene-rich cumulates, associated with the intrusion of calc-alkaline basic magmas at the base of the crust during an underplating event. The radiøgenic Sr compesition could be explained by infiltration -f an is tepically depleted melt (similar to the depleted SCS alkaline dykes) by an ${ }^{87} \mathrm{Sr} /{ }^{86} \mathrm{Sr}$ enriched compenent similar to Gb2 SCS calc-alkaline dykes.

We acknowledge A. F. Larios and J. G. del Tánago for their assistance with the electron microprobe analyses in the CAI of Microscopía Elecrónica (UCM); also B. A. Paterson for his collaboration with laser ablation microanalysis at the Department of Earth Sciences of the University of Bristol (UK), and J. M. F. Pérez and J. A. H. Jiménez from the CAI of Geocronología y Geoquímica (UCM) for their help in analysing samples by TIMS. The detailed revision by J. M. Cebriá and G. Ceuleneer greatly increase the quality of the manuscript. We are also indebte to G. Rogers for his revision of the English. This work is included in the objectives of, and supported by, the CGL-204-2515 project of the Ministerio de Educación y Ciencia of Spain.

\section{References}

Allègre, C. J. \& TurCotTe, D. L. 1987. Implications of a two-component marble-cake mantle. Nature, 323, 123-127.

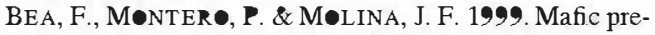
cursors, peraluminous granitoids, and late lamprophyres in the Avila batholith; a model for the generation of Variscan batholiths in Iberia. Joumal of Geology, 107, 399-419.

Bedini, R. M., Bodinier, J. L., Dautria, J. M. \& MøRTEN, L. 1997. Evolution of LILE-enriched small melt fractions in the lithospheric mantle: a case study from the East Af rica Rift. Earth and Planetary Science Letters, 153, 67-83.

Bedinier, J. L., VAsseur, G., Vernières, J., Dupuy, C. \& FABRIÉS, J. 1990. Mechanisms of mantle metasomatism: geochemical evidence from the
Lherz orogenic peridotite. Journal of Petrology, 31, $597-628$

BRUiJne, C. H. \& ANDRIESSEN, P. A. 2006. Interplay of in waplate tectonics and surface processes in the Sierra de Guadarrama (cenwal Spain) assessed by apatite fission track analyses. Physics and Chemistry of the Earth, 25, 555-563.

Carignan, J., Hil», P., Mevelle, G., Mørel, J. \& YEGHICHEYAN, D. 2001. Routine analyses of wace elements in geological samples using flow in jection and low pressure on-line liquid chromatography coupled to ICP-MS; a study of geochemical reference materials BR, DR-N, UB-N, AN-G and GH. Geostandards Newsletter, 25, 187-198.

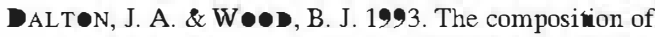
primary carbonate melts and their evolution through wallrock reaction in the mantle. Earth and Planetary Science Letters, 119, 511-525.

DEER, W. A., HowIE, R. A. \& Zussman, J. 1976. Rockforming Minerals: Volume 3, Sheet Silicates. Longman, Harlow.

DownES, H. 201. Formation and modification of the shallow sub-continental lithospheric mantle: a review of geochemical evidence from ul wamafic xenolith suites and tectonically emplaced ulwamafic massifs of western and cen Europe. Journal of Petrology, 42, 233-25e.

DownEs, H. \& DuPUy, C. 1987. Textural, isotopic and REE variations in spinel peridotite xenoliths, Massif Central, France. Earth and Planetary Science Letters, 82, 121-135.

Downes, H., EMBEy-IszTin, A. \& ThIRlWALl, M. F. 1992. Petrology and geochemiswy of spinel peridovite xenoiths from the western Pannonian Basin (Hungary): evidence for an association between enrichment and texture in the upper mantle. Contributions to Mineralogy and Petrology, 103, $277-286$.

Downes, H., Seghedi, I., SzakaCs, A. ET al. 1995. Perology and geochemistry of late Tertiary/Quaternary mafic alkaline magmatism in Romania. Lithos, 35, $65-81$.

Downes, H., Balaganskaya, E., BearD, A., Lifer@VICH, R. \& DEMAIFFE, D. 205. Petrogenetic processes in the ul ramafic, alkaline and carbonatitic magmatism in the Kola Alkaline Province: A review. Lithos, 85, 48-75.

Ducea, M. N. \& Saleeby, J. B. 1998. The age and origin of a thick mafic-ul wramafic keel from beneath the Sierra Nevada batholith. Contributions to Mineralogy and Petrology, 133, 169-185.

Escuder Viruete, J., Hernáiz, P. P., Valverde, P., R@DíGUEZ, R. \& DUNnING, G. 1998. Variscan syncollisional extension in the Iberian Massif: suctural, metamorphic and geochronological evidence from the Somosierra sector of the Sierra de Guadarrama (Cen mal Iberian Zone, Spain). Tectonophysics, 290, 87-109.

ESPERANÇA, S. \& HølløWAy, J. R. 1987. On the origin of some mica-lamprophyres; experimental evidence from mafic minette. Contributions to Mineralogy and Petrology, 95, 207-216.

FÉménias, O., Coussaert, N., Bingen, B., Whiteh use, M., Mercier, J. C. C. \& Demaiffe, D. 
203. A Permian underplating event in late- to post-orogenic tectonic setwing. Evidence from the mafic-ulmamafic layered xenoliths from Beaunit (French Massif Central). Chemical Geology, 199, 293-315.

FERnÁNDEZ-SuÁrez, J., JefFries, T. E., Whiteh@use, M. J., AREnas, R. \& Villaseca, C. 2006. A U-Pb study of zircons from lower crustal xenoliths of the Spanish Cen ral System: a record of Iberian lithospheric evolution from the Neoproterozoic to the Triassic. Joumal of Geology, 114, 471-483.

Føley, S. F., Jacksøn, S. E., Fryer, B. J., GREen-ugh, J. D. \& Jenner, G. A. 1996. Trace element partition coefficients for clinopyroxene and phlogopite in an alkaline lamprophyre from Newfoundland by LAM-ICP-MS. Geochimica et Cosmochimica Acta, 60, 629-638.

FREY, F. A. \& PRINZ, M. 1978. Ul ramafic inclusions from San Carlos, Arizona; petrologic and geochemical data bearing on their petrogenesis. Earth and Planetary Science Letters, 38, 129-176.

Galinde, C., Huertas, M. J. \& Caseuet, C. 1994. Cronología Rb-Sr y K-Ar de diques de la Sierra de Guadarrama (Sistema Central Español). Geogaceta, 16, 23-26.

GARRIDe, C. J. \& BeDINIER, J. L. 1999. Diversity of mafic rocks in the Ronda Peridotite; evidence for pervasive melt-rock reaction during heating subconinental lithosphere by upwelling asthenosphere. Journal of Petrology, 40, 729-754.

Green, D. H. \& WAllace, M. E. 1988. Mantle metasomatism by ephemeral carbonate melts. Nature, 336, 459-462.

Grégeire, M., Møine, B. N., O’Reilly, S. Y., Cottin, J. Y. \& Giret, A. 20e0. Trace element residence and partitioning in mantle xenoliths metasomatize by highly alkaline, silicate- and carbonaterich melts (Kerguelen Islands, Indian Ocean). Journal of Petrolo:y, 41, 477-509.

Grégøire, M., Bell, D. R. \& LE R•eX, A. P. 2003. Garnet lherzolites from the Kaapvaal craton (South Africa): wace element evidence for a metasomatic history. Journal of Petrology, 44, 629-657.

HART, S. R. \& DUNN, T. 1993. Experimental cpx/melt partitioning of 24 wace elements. Contributions to Mineralogy and Petrology, 113, 1-8.

I॰N॰V, D. A. 1998. Trace element composition of mantlederived carbonates and coexisting phases in peridotite xenoliths from alkali basalts. Journal of Petrology, 39, 1931-1941

IøN•V, D. A. \& HøFMANN, A. W. 1995. Nb-Ta-rich mantle amphiboles and micas: implications for subduction-related metasomatic element fractionations. Earth and Planetary Science Letters, 131, 341-356.

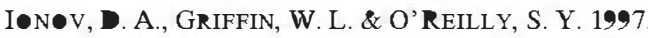
Volatile-bearing minerals and lithophile trace elements in the upper mantle. Chemical Geology, 141, 153-184.

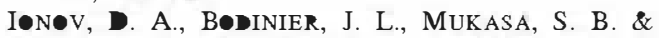
ZANETTI, A. 202a. Mechanisms and sources of mantle metasomatism: ma jor and race element compositions of peridotite xenoliths from Spitsbergen in the context of numerical modelling. Joumal of Petro$\log y, 43,2219-2259$

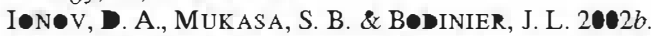
$\mathrm{Sr}-\mathrm{Nd}-\mathrm{Pb}$ isotopic compositions of peridotite xenoliths from Spitsbergen: numerical modelling indicates $\mathrm{Sr}-\mathrm{Nd}$ decoupling in the mantle by melt percolation metasomatism. Journal of Petrology, 43, 2261-2278.

IRVING, A. J. \& FREY, F. A. 1984. Trace element abuudances in megacrysts and their host basalts; conswaints on partition coefficients and megacryst genesis. Geochimica et Cosmochimic Acta, 48, 12-1221.

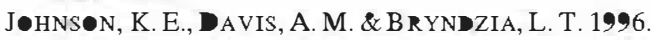
Conwasting styles of hydrous metasomatism in the upper mantle: an ion microprobe investigation. Geochimica et Cosmochimica Act a, 60, 1367-1385.

KEMPT N, P. D. 1987. Mineralogical an geochemical evidence for differing styles of metasomatism in spinel lherzolite xenoliths: enriched mantle source regions of basalts. In: MENZIES, M. \& HAwkES. WORTH, C. J. (eds) Mantle Metasomatism. Academic Press, New York, 45-89.

KEMPT@N, P. D., HAWKESWORTH, C. J., López-Escobar, L., Pears@n, D. G. \& Ware, A. J., 1999. Spinel \pm garnet lherzolite xenoliths from Pali Aike, Part 2: wace element and isotopic evidence on the evolution of lithospheric mantle beneath southern Patagonia. In: GURney, J., GURney, J. L., PASCD, M. D. \& RICHARDS, S. H. (eds.) The B. J. Dawson Volume, Proceedings of the 7 th International Kimberlite Conference. Red Roof Design, Cape Town, 415-428.

Klemme, S., VAn DeR LaAn, S. R., Føley, S. F. \& GÜNTHER, D. 1995. Experimentally determined trace and minor element partitioning between clinopyroxene and carbonatite melt under upper mantle conditions. Earth and Planetary Science Letters, 133, 439-448.

latøurkette, T., Hervig, R. L. \& Høll@Way, J. R. 1995. Trace element partitioning between amphibole, phlogopite, and basanite melt. Earth and Planetary Science Letters, 135, 13-30

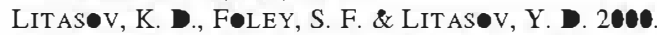
Magmatic modification and metasomatism of the subcontinental mantle beneath the Vitim volcanic field (East Siberia): evidence from wace element data on pyroxenite and peridote xenoliths from Miocene picrobasalt. Lithos, 54, 83-114.

Mattielli, N., Weis, D., Scates, J. S. er al. 1999. Evolution of heterogeneous lithospheric mantle in a plume environment beneath the Kerguelen Archipelago. Journal of Petrology, 40, 1721-1744.

MCD•N@UGH, W. F. \&SUn, S. S. 1995. The composition of the Earth. Chemical Geology, 120, 223-253.

MerCier, J. C. C. 1980. Single-pyroxene thermobaromey. Tectonophysics, 70, 1-37.

MINARIK, W. G. 1998. Complications to carbonate melt mobility due to the presence of an immiscible silicate melt. Joumal of Petrology, 39, 1965-1973.

NAV•N, O. \& St LPER, E. 1987. Geochemical consequences of melt percolation; the upper mantle as a chromatographic column. Joumal of Geology, 95, $285-307$ 
Nimis, P. \& UlMER,P. 1998. Clinopyroxene geobarome of magmatic rocks Part 1: An expanded structural geobarometer for anhydrous and hydrous, basic and ul rabasic systems. Contributions to Mineralogy and Petrology, 133, 122-135.

O'Reilly, S. Y., GRiffin, W. L. \& Ryan, C. G. 1991 Residence of race elements in metasomazel spinel lherzolite xenoliths; a proton-microprobe study. Contributions to Mineralogy and Petrology, 109, 98-113.

OREJANA, D., Villaseca, C. \& PATERs N, B. A. 206 Geochemisty of pyroxenitic and hormblenditic xenoliths in alkaline lamprophyres from the Spanish Cenwal System. Lithos, 86, 167-196.

OTtEN, M. T. 1984. The origin of brown homblende in the Artfjaellet gabbro and dolerites. Contributions to Mineralozy and Petrolosy, 86, 189-199.

PAWLEY, A. R. 2003. Chlorite stability in mantle peridoite: the reaction clinochlore + enstate $=$ forsterite + pyrope $+\mathrm{H}_{2} \mathrm{O}$. Contributions to Mineralogy and Petrology, 144, 449-456.

PAWLEY, A. R. \& W $\mathbf{~ D , ~ B . ~ J . ~ 1 9 9 5 . ~ T h e ~ h i g h ~ p r e s s u r e ~}$ stability of talc and $10 \dot{A}$ phase: Potential storage sites for $\mathrm{H}_{2} \mathrm{O}$ in subduction zones. American Mineralogist, 80, 998-1003.

Perini, G., Cebriá, J. M., Lépez-Ruiz, J. M. \& DڤBlas, M. 204. Permo-Carboniferous magmatism in the variscan belt of Spain and France: implications on mantle sources. In: WILS N, M., NEUMANN, E. R., Davies, G. R., Timmerman, M. J., HeereMANS, M. \& LARSEN, B. (eds) Penno-Carboniferous Magmatism and Rifting in Europe. Geological Society, London, Special Publications, 223, 415-438.

Reyes, J., Villaseca, C., Barber•, L., Quejide, A. J. \& SAntos, J. F. 1997. Descripción de un método de separación de $\mathbf{R} b, \mathrm{Sr}, \mathrm{Sm}$ y $\mathrm{N} \mathbf{d}$ en rocas silicatadas para estudios isotópicos. In: Actas del I Congreso Ibérico de Geoquímica. CEDEX, Soria, 46-55.

R॰CK, N. M. S. 1991. Lamprophyres. Blackie, Glasgow.

Rosenbaum, M. J., Wils@N, M. \& Downes, H. 1997 Enrichment of the Pannonian Carpathian mantle: $\mathrm{Pb}-\mathrm{Sr}-\mathrm{Nd}$ isotope and race element conswaints. Journal of Geophysical Research, 102, 14947-14961.

Scarr@W, J., Bea, F., Monter •, P., Mølina, J. F. \& Vaughan, A. P. M. 2006. A precise late Pennian ${ }^{4} \mathrm{Ar} /{ }^{39} \mathrm{Ar}$ age for Cenwal Iberian camptonitic lamprophyres. Geologic Act 4, 451-459.

SHAW, C. S. J. 2044. The temporal evolution of three magmatic systems in the West Eifel volcanic field, Germany. Journal of Volcanology and Geothermal Research, 131, 213-240

Skulski, T., Minarik, W. \& Wats $\bullet$, E. B. 1994 High-pressure experimental race-element partitioning between clinopyroxene and basaltic melts. Chemical Geology, 117, 127-147.

Sun, S. S. \& MCDenøugh, W. F. 1989. Chemical and isotopic systematics of oceanic basalts; implications for mantle composition and processes. In: SAUNDERS, A. D. \& NoRRY, M. J. (eds) Magmatism in Ocean Basins. Geological Society, Special Publications, 42, 313-345.
Vaselli, O., Downes, H., Thirlwall, M. F. er al. 1995. Ulwamafic xenoliths from Plio-Pleistocene alkali basalts from the Eastern Transylvanian Basin: depleted mantle enriched by vein metasomatism. Joumal of Petrology, 38, 23-54.

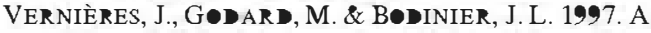
plate model for the simulation of trace element fracionation during partial melting and magmas wansport in the Earth's upper mantle. Joumal of Geophysical Research, 102, 24771-24784.

Villaseca, C., Downes, H., Pin, C. \& Barber •, L. 1999. Nature and composition of the lower continental crust in Cen Spain and the granulite-granite linkage: inferences from granulitic xenoliths. Journal of Petrology, 40, 1465-1496.

Villaseca, C., Orejana, D., Pin, C., Lépez García, J. A. \& ANDonAEgUI, P. 2004. Le magmatisme basique hercynien et post-hercynien du Système Central Espagnol: essai de caractèrisation des sources manteliques. Comptes Rendus de Géosciences, 336, 877-888.

Wilshire, H. G., 1987. A model of mantle metasomaism. In: MorRIS, E. M. \& PASTERIS, J. D. (eds) Mantle Metasomatism and Alkaline Magmatism. Geological Society of America, special papers, 215, 47-6e.

WILs@N, M. 1989. Igneous Petrogenesis: a Global Tectonic Approach. Unwin Hyman, Boston, MA.

Witt-Eickschen, G., Seck, H. A., MeZger, K., EgGins, S. M. \& AltherR, R. 202. Lithospheric mantle evolution beneath the Eifel (Germany): constraints from $\mathrm{Sr}-\mathrm{Nd}-\mathrm{Pb}$ isotopes and wace element abundances in spinel peridote and pyroxenite xenoliths. Journal of Petrology, 44, 1077-1095.

Weelley, A. R. \& KeMPE, D. R. C. 1989. Carbonatites: nomenclature, average chemical compositions, and element distribution. In: BELL, K. (ed.) Carbonatites: Genesis and Evolution. Unwin Hyman, London, 1-14.

Xu, X., O'Reilly, S. Y., Griffin, W. L. \& ZH•u, X. 203. Enrichment of upper mantle peridotite: perological, wace element and isotopic evidece in xenoliths from SE China. Chemical Geology, 198, $163-188$

XU, Y. G. 2022. Evidence for crustal components in the mantle and conswaints on crustal recycling mechanisms: pyroxenite xenoliths from Hannuoba, North China. Chemical Geology, 182, 31-322.

XU, Y. G. \& BøiniER, J. L. 204. Con rasing enrichments in high- and low-temperature mantle xenoliths from Nushan, eastern China: results of a single metasomatic event during lithospheric accretion. Journal of Petrology, 45, 321-341

Yaxley, G. M., GreEn, D. H. \& Kamenetsky, V. 1998. Carbonatite metasomatism in the southeastern Auswalia lithosphere. Joumal of Petrology, 39, 1917-1930.

Zanetti, A., VannuCCi, R., Bett aZZi, P., Oberti, R. \& OTT LINI, L. 1996. Infilmation metasomatism at Lherz as monitore by systematic ion-microprobe investigations close to a homblendite vein. Chemical Geology, 134, 113-133. 